\title{
Glutamate Signaling via the AMPAR Subunit GluR4 Regulates Oligodendrocyte Progenitor Cell Migration in the Developing Spinal Cord
}

\author{
${ }^{\circledR}$ Melanie Piller,* Inge L. Werkman,* Evan A. Brown, Andrew J. Latimer, and ${ }^{\circledR}$ Sarah Kucenas \\ Department of Biology, University of Virginia, Charlottesville, Virginia 22904
}

\begin{abstract}
Oligodendrocyte progenitor cells (OPCs) are specified from discrete precursor populations during gliogenesis and migrate extensively from their origins, ultimately distributing throughout the brain and spinal cord during early development. Subsequently, a subset of OPCs differentiates into mature oligodendrocytes, which myelinate axons. This process is necessary for efficient neuronal signaling and organism survival. Previous studies have identified several factors that influence OPC development, including excitatory glutamatergic synapses that form between neurons and OPCs during myelination. However, little is known about how glutamate signaling affects OPC migration before myelination. In this study, we use in vivo, timelapse imaging in zebrafish in conjunction with genetic and pharmacological perturbation to investigate OPC migration and myelination when the GluR4A ionotropic glutamate receptor subunit is disrupted. In our studies, we observed that gria4a mutant embryos and larvae displayed abnormal OPC migration and altered dorsoventral distribution in the spinal cord. Genetic mosaic analysis confirmed that these effects were cell-autonomous, and we identified that voltage-gated calcium channels were downstream of glutamate receptor signaling in OPCs and could rescue the migration and myelination defects we observed when glutamate signaling was perturbed. These results offer new insights into the complex system of neuron-OPC interactions and reveal a cell-autonomous role for glutamatergic signaling in OPCs during neural development.
\end{abstract}

Key words: glutamate signaling; myelin; oligodendrocyte; oligodendrocyte progenitor cell; zebrafish

\section{Significance Statement}

The migration of oligodendrocyte progenitor cells (OPCs) is an essential process during development that leads to uniform oligodendrocyte distribution and sufficient myelination for central nervous system function. Here, we demonstrate that the AMPA receptor (AMPAR) subunit GluR4A is an important driver of OPC migration and myelination in vivo and that activated voltage-gated calcium channels are downstream of glutamate receptor signaling in mediating this migration.

\section{Introduction}

During development, oligodendrocyte progenitor cells (OPCs) migrate from their ventral spinal cord origin and distribute throughout the CNS, and a subset differentiates into mature oligodendrocytes that myelinate axonal segments (Barres and Raff, 1999; Kucenas et al., 2008; Emery, 2010; de Faria et al., 2019;

Received Oct. 2, 2020; revised Apr. 28, 2021; accepted May 3, 2021.

Author contributions: M.P. and S.K. designed research; M.P., I.L.W., and S.K. performed research; E.A.B. and A.J.L. contributed unpublished reagents/analytic tools; M.P. and I.L.W. analyzed data; M.P., I.L.W., and S.K. wrote the paper.

This work was supported by the Arnold and Mabel Beckman Foundation (M.P.), The Owens Family Foundation (S.K.), and National Institutes of Health Grants NS072212 (to S.K.) and NS107525 (to S.K.). We thank members of the Kucenas Lab, past and present, for valuable discussions, Dr. Alexandria Hughes and Dr. Laura Fontenas for technical help with glutamate uncaging in vivo, and Lori Tocke for zebrafish care.

${ }^{*}$ M.P. and I.L.W. are co-first authors.

The authors declare no competing financial interests.

Correspondence should be addressed to Sarah Kucenas at sk4ub@virginia.edu.

https://doi.org/10.1523/JNEUROSCI.2562-20.2021

Copyright $\odot 2021$ the authors
Mitew et al., 2014; Nave and Werner, 2014; Marisca et al., 2020). During this process, OPCs interact extensively with each other and their environment, and are influenced by a variety of signals, including growth factors, chemokines, neurotransmitters, and extracellular matrix components (Kirby et al., 2006; Frost et al., 2009; Hughes et al., 2013; Sanchez-Rodriguez et al., 2018; Suzuki et al., 2019). Previously, we identified adenosine signaling via neuronal A2A receptors as an upstream regulator of OPC migration (Fontenas et al., 2019). In this study, we sought to extend those findings and identify an axonally-derived cue that modulates OPC migration during development.

Previous studies demonstrate that neuronal signaling influences OPC development via various mechanisms, including OPCexpressed ionotropic AMPA receptors (AMPARs; Bergles et al., 2000; Lin and Bergles, 2004). The activation of OPC AMPARs can affect cellular processes by facilitating membrane depolarization and subsequent activation of voltage-gated calcium channels, resulting in $\mathrm{Ca}^{2+}$ influx (Gudz et al., 2006; Paez et al., 2010; Giesen et al., 2020). In vivo, neuronal activity affects oligodendrocyte axon 
Table 1. Zebrafish lines

\begin{tabular}{|c|c|c|}
\hline Name & Source & Description \\
\hline$A B *$ & & Wild type \\
\hline $\operatorname{Tg}(\text { olig2:dsred) })^{\text {vu19 }}$ & Shin et al. (2003) & Red fluorescent line that labels olig $2^{+}$motor neurons and OLCs \\
\hline $\operatorname{Tg}(\text { olig2:egfp) })^{\text {vu12 }}$ & Shin et al. (2003) & Green fluorescent line that labels olig2 ${ }^{+}$motor neurons and OLCs \\
\hline $\operatorname{Tg}(m b p: e g f p-(A A X)$ & Almeida et al. (2011) & Green fluorescent membrane line that labels $m b p^{+}$myelinating cells \\
\hline $\operatorname{Tg}(\text { sox } 10: \text { megfp })^{s / 3}$ & Kirby et al. (2006) & Green fluorescent membrane line that labels sox $10^{+}$glia and neural crest cells \\
\hline Gt(foxd3:m(herry) $)^{c t 110 R}$ & Hochgreb-Hägele and Bronner (2013) & Red fluorescent gene-trap line that labels foxd $3^{+}$neural crest cells and peripheral glia \\
\hline $\operatorname{Tg}(X \mid a . T u b b: d s r e d)$ & Peri and Nüsslein-Volhard (2008) & Red fluorescent line that labels tubulin ${ }^{+}$neurons \\
\hline gria4a $a^{\text {uva43 }}$ & This article & 13-bp insertion resulting in frameshift and early stop codon (see Fig. 1D) \\
\hline $\operatorname{Tg}\left(\right.$ sox 10:SF-iGluSnFR) ${ }^{u v a 56}$ & This article & i-GluSnFr line that labels sox $10^{+}$glia with a cell membrane glutamate sensor \\
\hline
\end{tabular}

selection for myelination and stabilization of myelin sheath formation (Hines et al., 2015; Koudelka et al., 2016), and neuronal signaling is also implicated in OPC proliferation (Barres and Raff, 1999; Gibson et al., 2014) and survival (Kougioumtzidou et al., 2017). Several studies have reported that AMPAR-mediated $\mathrm{Ca}^{2+}$ influx activates OPC migration in vitro (Gudz et al., 2006; Paez et al., 2010; Harlow et al., 2015), but this effect has not been described in vivo (Harlow et al., 2015; Kougioumtzidou et al., 2017) and currently, there is no consensus on how AMPARs affect OPC migration in vivo.

In addition to AMPAR-mediated activation of voltage-gated calcium channels, extracellular $\mathrm{Ca}^{2+}$ influx can occur via calcium-permeable AMPARs. There are eight AMPAR subunit genes encoded in the zebrafish genome, compared with four in mammals, because of a whole-genome duplication event in the evolutionary history of teleost fish (Meyer and Schartl, 1999; Sobolevsky et al., 2003). Of these eight zebrafish AMPAR subunit genes, gria $2 a$ and gria $2 b$ are calcium impermeable (Kung et al., 2001; Sobolevsky et al., 2003). The remaining six AMPAR subunit genes, including gria $4 a$, which encodes GluR4A, are calciumpermeable (Kung et al., 2001). The calcium permeability of an AMPAR is determined by the permeability of each of its subunits (Burnashev, 1998), and calcium-permeable AMPARs are a major source of $\mathrm{Ca}^{2+}$ influx in OPCs (Ge et al., 2006). This mechanism of $\mathrm{Ca}^{2+}$ influx via calcium-permeable AMAPRs has been studied extensively in the context of excitotoxicity (Feldmeyer et al., 1999; Higuchi et al., 2000; Lee et al., 2001; Isaac et al., 2007; Evonuk et al., 2020) and extracellular $\mathrm{Ca}^{2+}$ influx can cause a wide array of cellular effects in OPCs, including changes in proliferation and differentiation (Chen et al., 2018), cell death and survival (Danesi et al., 2019), myelination (Krasnow et al., 2018), and cell morphology and migration (Gudz et al., 2006; Paez et al., 2010; Harlow et al., 2015). In primary rat cultures, OPCs and immature oligodendrocytes highly express GluR4, while GluR4 expression and $\mathrm{Ca}^{2+}$ influx was decreased in mature, myelin basic protein (MBP)expressing oligodendrocytes, indicating that AMPAR subunit composition and downstream $\mathrm{Ca}^{2+}$ influx may control the onset of myelination (Itoh et al., 2002). However, the relationship between AMPAR subunit composition and downstream $\mathrm{Ca}^{2+}$ influx with regard to OPC migration in vivo, is not fully understood (Harlow et al., 2015).

Here, using in vivo, time-lapse imaging coupled with a new gria $4 a$ CRISPR mutant, we demonstrate that the migration and myelination patterns of spinal cord OPCs and oligodendrocytes are disrupted in the presence of perturbed GluR4A. Our results highlight, in vivo, a role for AMPAR activation downstream of neuronal activity and identify the GluR4A subunit as a regulator of OPC migration before myelination during vertebrate development.

\section{Materials and Methods}

\section{Zebrafish}

The use of all animals in this study was approved by the University of Virginia Institutional Animal Care and Use Committee. The zebrafish lines used were: $\mathrm{AB} *$ (wild type), $\operatorname{Tg}(\text { olig2:dsred })^{\text {vu19 }}, \operatorname{Tg}(\text { olig2:egf })^{\text {vu12 }}$ (Shin et al., 2003), Tg(mbp:egfp-CAAX) (Almeida et al., 2011), Tg(sox10: $m e g f p)^{s l 3}$ (Smith et al., 2014), Gt(foxd3:mCherry) ${ }^{c t 110 R}$ (Hochgreb-Hägele and Bronner, 2013), Tg(Xla.Tubb:dsred) (Peri and Nüsslein-Volhard, 2008), Tg(sox10:SF-iGluSnFR) ${ }^{\text {uva }}$, and gria $4 a^{\text {uva } 43}$ (Table 1). To visualize individual oligodendrocyte lineage cells (OLCs), one-cell $\mathrm{gria}_{4} \mathrm{a}^{+/+}$, gria $4 a^{+/ \text {uva } 43}$ and gria4a $a^{\text {uva43/uva43 }}$ embryos were transiently injected with $2 \mathrm{nl}$ of $20 \mathrm{ng} / \mu \mathrm{l}$ mbp:egfp-CAAX (Almeida et al., 2011). Embryos were incubated at $28.5^{\circ} \mathrm{C}$ in egg water, and phenylthiourea (PTU; $0.004 \%$ ) was used to reduce pigment formation for imaging (Karlsson et al., 2001). Embryos of either sex were used in all experiments (Kimmel et al., 1995), and ages are listed in hours postfertilization (hpf) or days postfertilization (dpf).

Generation of gria4a ${ }^{\text {uva } 43 / \mathrm{uva} 43}$ mutants and the $\mathrm{Tg}(\operatorname{sox} 10: \mathrm{SF}-\mathrm{iGluSnFR})$ line

Guide RNA targeting gria4a (TGAGAGGTTCATGCACGGCG) was designed using CHOPCHOP (Labun et al., 2018) and the protocol described by Gagnon et al. (2014). The 3' constant and 5' gria4a-specific oligonucleotides were combined as described by Nakayama et al. (2014), and the sgRNA transcribed using the Ambion Megascript T7 kit. An injection of $2 \mathrm{nl}$ of the sgRNA $(200-400 \mathrm{ng} / \mu \mathrm{l})$ with Cas9 protein $(500 \mathrm{ng} / \mu \mathrm{l})$ was performed into one-cell stage $A B *$ embryos. Once the injected fish reached adulthood, they were bred to $\mathrm{AB} *$ wildtype adults and DNA isolated from a pool of 8 embryos was amplified with PCR using the primers TTAGTGTGTCAGCATTTGGGAC and TAGTTTGATCACCATCGGTTTG, then sequenced to identify potential founders. $T g$ (olig2:dsred) and $T g(m b p$ :egfp-CAAX) were crossed with the injected adult fish (F0) with a germline mutation in gria4a to produce stable heterozygote mutants. These fish (F1) were raised to adulthood, and fin clipping and sequencing was used to determine each mutation. We identified a 13-bp insertion resulting in a premature stop codon, and fish bearing this mutation were crossed to produce all subsequent generations. This 13-bp insertion produces a restriction site for MslI not found in the wild-type gria $4 a$ gene, and restriction digest of the amplified fragment followed by agarose gel electrophoresis on a $1.5 \%$ agarose gel was used for genotyping. This digest produces two bands at 149 and $75 \mathrm{bp}$ for the mutant gene and does not affect the 224-bp wildtype fragment. All three bands are visible following digestion of heterozygous DNA. Whenever possible, genotyping was performed after analyzing results to allow for blind analysis of all studies.

$\mathrm{Tg}$ (sox10:SF-iGluSnFR) fish were generated via the Tol2kit Gatewaybased cloning system (Kwan et al., 2007). Vectors used for making the Gateway expression constructs were p5E-sox10(-4.9) (Carney et al., 2006), pME-SF-iGluSnFR.A184V (Marvin et al., 2018), p3E-poly A (Kwan et al., 2007), and the destination vector pDesTol2CG2 (Kwan et al., 2007). All three expression vectors were inserted into the pDesTol2CG2 destination vector via LR reactions (Kwan et al., 2007). Resulting LR plasmids were amplified and sequenced to confirm appropriate insertion of each expression construct. After sequence confirmation, the plasmid was injected into one-cell stage $A B *$ embryos at a 
concentration of $25 \mathrm{ng} / \mu \mathrm{l}$ with an equal concentration of Tol2 transposase mRNA. Fish expressing the pDesTol2CG2 heart reporter and SFiGluSnFR fluorescence were raised to adulthood and were crossed to $\mathrm{AB} *$ adults to screen for founders (Kawakami, 2004).

\section{Cell transplantation}

Donor embryos were injected with ThermoFisher Dextran Cascade Blue dye $(3000 \mathrm{MW})$ immediately following fertilization at the one-cell stage. All embryos were manually dechorionated at $4 \mathrm{hpf}$ in $2 \%$ agarose-lined dishes and flamed pipettes were used to transfer dechorionated embryos. Transplantation was performed using the Eppendorf CellTram vario transplantation rig, with $\sim 30$ cells transferred from each donor embryo and inserted into the expected spinal cord region of the 4- to 5-hpf host embryo according to fate mapping (Kimmel et al., 1995). Transplantation was performed in $1 \times$ Danieau with $1 \times$ penicillin-streptomycin, and hosts were incubated at $28.5^{\circ} \mathrm{C}$ in $0.3 \times$ Danieau with $1 \times$ penicillin-streptomycin following the procedure. The hosts were later imaged as described below and both donor and host embryos were kept and genotyped as described above.

\section{Chemical treatments}

Drug treatments [NBQX, ( \pm )-Bay K 8644] were performed by adding a working solution of each drug, dissolved in $1 \%$ dimethylsulfoxide (DMSO), to 96-well plates containing one embryo per well. ( \pm )-Bay $\mathrm{K}$ 8644 was applied at a treatment concentration of $5 \mu \mathrm{M}$ as previously described (Koleilat et al., 2019). A treatment of 1\% DMSO was used as a control for each drug.

\section{Confocal imaging}

All confocal images were taken with a $40 \times$ water objective $(\mathrm{NA}=1.1)$ mounted on a motorized Zeiss AxioObserver Z1 microscope equipped with a Quorum WaveFX-XI (Quorum Technologies Inc.) or Andor CSU-W (Andor Oxford Instruments) spinning disk confocal system. Embryos used for imaging were anesthetized with $0.01 \%$ 3-aminobenzoic acid ester (Tricaine) and mounted in $0.8 \%$ low-melting-point agarose in $35 \mathrm{~mm}$ glass-bottomed Petri dishes, then immersed in egg water with PTU $(0.004 \%)$. For drug treatment experiments, the immersion during imaging contained the same dosage administered in the 96-well plate, described above. In time-lapse experiments, images were taken every $10 \mathrm{~min}$ unless otherwise noted. Images were processed with MetaMorph and ImageJ softwares, and adjustments were limited to levels and contrast. Cell migration tracking was performed on time-lapses with images every $20 \mathrm{~min}$, for consistency, using the Image Manual Tracking plugin, as described below. Myelin quantification was performed through the $\mathrm{z}$-stack of each image using the measurement feature of ImageJ.

\section{Cell tracking}

The ImageJ plugin Manual Tracking was used to determine speed and distance traveled of migrating cells. For each cell, a track was created by clicking the center of the cell body at each time point in a time-lapse. Distance migrated was calculated by summing the "distance" output, and migration speed was calculated by averaging the "velocity" output, both scaled to $\mu \mathrm{m}$. Further documentation can be found on https:// imagej.nih.gov/ij/plugins/track/track.html.

\section{Myelin measurement}

The "measure" feature of ImageJ was used to quantify the length of myelin internodes. To do this, the line tool was used to mark myelin sheaths appearing in either individual z-planes or z-stacks of images of $T g(m b p$ : egfp-CAAX) embryos or embryos transiently injected with the mbp:egfp$C A A X$ construct (see figure legends). The results were compiled and scaled to $\mu \mathrm{m}$.

\section{Photo-uncaging glutamate}

We bathed embryos and larvae in $1 \mu \mathrm{m}$ MNI-glutamate (Tocris catalogue number 1490; $25 \mathrm{~mm}$ stock solution in DMSO) $1 \mathrm{~h}$ before imaging. An equal amount of DMSO (0.4\%) was used as a vehicle control. During treatment, larvae were kept in the dark as much as possible and we mounted larvae for live imaging as described above. We performed focal uncaging of MNI-glutamate with a 404-nm laser focused to a circular region of interest $\left(50-\mu \mathrm{m}^{2}\right.$ surface, $\sim 20-40 \mu \mathrm{m}$ from sox $10^{+}$OPC membranes) in the center of the acquired z-stack, followed by time-lapse imaging for $1 \mathrm{~min}$, imaging every $32 \mathrm{~ms}$, or for $2 \mathrm{~h}$, imaging every minute.

\section{In situ hybridization}

Embryos and larvae used were fixed in $4 \%$ paraformaldehyde (PFA) overnight at $4^{\circ} \mathrm{C}$ and stored in $100 \%$ methanol. In situ RNA hybridization was used to localize the expression of gria $4 a$ in the zebrafish spinal cord. The primers used to produce the probes were as follows: forward primer, ACATCTCTCGCAGAGGCAAT; reverse primer, GGGCTTGGAGAAATCAATCA. Probes were prepared with DIG labeling, as previously described (Fontenas et al., 2019). After in situ hybridization, embryos and larvae were embedded in $1.5 \%$ agarose and saturated with $30 \%$ sucrose in distilled water, then frozen in 2methylbutane cooled by immersion in liquid nitrogen. We produced $25 \mu \mathrm{m}$ cross-sections of the embryos/larvae with a cryostat microtome, then immersed in $75 \%$ glycerol and imaged the slides with the $40 \times$ water objective $(\mathrm{NA}=1.1)$ on a Zeiss AxioObserver microscope. Images were imported to Adobe photoshop, and adjustments were limited to levels, contrast, and cropping.

\section{Immunohistochemistry}

Embryos and larvae were fixed in 4\% PFA for $1 \mathrm{~h}$, then embedded in $1.5 \%$ agarose and saturated with $30 \%$ sucrose in distilled water. Then, they were frozen in 2-methylbutane cooled by immersion in liquid nitrogen. We produced $25 \mu \mathrm{m}$ cross-sections of the embryos/larvae with a cryostat microtome. Antibodies used were as following: rabbit antiSox10 (1:1000; Binari et al., 2013) and chicken anti-GFP (Aves Lab; 1:500). Fluorescent detection of antibody labeling was performed using Alexa Fluor 555 donkey anti-rabbit (1:500) and Alexa Fluor 488 donkey anti-chicken (1:500). Slides were imaged using a $40 \times$ oil objective $(\mathrm{NA}=1.1)$ on a Zeiss AxioObserver ZI microscope. Images were imported to ImageJ, and adjustments were limited to levels, contrast, and cropping. For immunohistochemistry following in situ hybridization, whole mount in situ hybridization was performed as described above and then embryos or larvae were embedded in agarose, cryosectioned, and then immunohistochemistry was performed as described.

\section{Cell dissociation for flow cytometry}

For cell dissociation, $72 \mathrm{hpf}$ larvae with transgenes $\operatorname{Tg}$ (olig2:dsred); $\operatorname{Tg}$ (sox10: megfp) for OPCs, $T g($ sox10:megfp);Gt(foxd3:mCherry) for Schwann cells, and $\operatorname{Tg}(n b t: d s r e d)$ for neurons, were chilled in egg water on ice, then the heads were removed using a scalpel. The cells were dissociated according to a previously published method (Zhu et al., 2019). Briefly, the trunks were immersed in calcium-free Ringer's solution with $2.5 \mathrm{~mm}$ EDTA and rocked for $15 \mathrm{~min}$ at $4^{\circ} \mathrm{C}$, then washed three times with chilled Dulbecco's PBS (DPBS) and transferred with $100 \mu \mathrm{l}$ D-PBS into microcentrifuge tubes. The trunk pieces were broken up using a pellet pestle followed by the addition of $30 \mu \mathrm{l}$ Liberase TM. The samples were incubated for $15 \mathrm{~min}$ at $28.5^{\circ} \mathrm{C}, 1$ $\mathrm{ml}$ Trypsin with 5\% EDTA was added, then the samples were incubated again for $15 \mathrm{~min}$ at $28.5^{\circ} \mathrm{C}$. The samples were pipetted into 5-ml D-PBS with $1 \% \mathrm{BSA}$, then filtered by passing through a $40 \mu \mathrm{m}$ cell strainer, and a syringe plunger was used to gently mash the samples into a Petri dish. The sample was passed through a new $40 \mu \mathrm{m}$ cell strainer and transferred to a microcentrifuge tube, then washed twice with D-PBS with $1 \%$ BSA. Three samples of cells $(1750 \pm 750)$ of each type were used for RNA-sequencing. Total RNA was extracted using the RNeasy Micro kit (QIAGEN), then cDNA was prepared using Smart-Seq v4 Ultra Low Input RNA kit for sequencing (Takara). Library preparation was performed using the NEB Next Ultra II DNA Library Prep kit for Illumina (NEB).

\section{RNA-seq analysis}

RNA-seq analysis was performed following previously published methods (Zhu et al., 2019). Briefly, abundance of transcripts from RNA-seq datasets were imported into the DESeq2 pipeline using the $\mathrm{R}$ package tximport (Love et al., 2014; Soneson et al., 2015). R package AnnotationDbi was used to acquire ENTREZ IDs and Gene Symbols 
(Pagès et al., 2020). Normalized FPKM counts were generated using the fpkm() function in the DESeq2 package (Love et al., 2014). Differentially expressed genes were analyzed using the DESeq2 package (abs (log2FoldChange) $>1$ \&padj $<0.1$; Love et al., 2014). Lists of differentially expressed genes were then used to perform functional analysis using the clusterProfiler package (Yu et al., 2012). Genes with padj $==$ NA were removed to exclude genes with low counts. Raw sequencing data are available on GEO: [https://www.ncbi.nlm.nih.gov/geo/query/ acc.cgi?acc=GSE174486]. Transcript abundance files are available on Mendeley Data: [https://data.mendeley.com/datasets/cpfwssf2ns/2].

\section{$R T-P C R$}

mRNA was extracted and cDNA synthesized as described previously (Peterson and Freeman, 2009) with the use of RNAeasy Mini kit (QIAGEN) and High-capacity cDNA Reverse Transcription kit (AppliedBiosystems) according to manufacturer instructions. Equal amounts of mRNA were used for cDNA synthesis, and RT-PCR was performed using GoTaq green mastermix (Promega). Primers used were: gria4a: forward, CAAGGACTCGGGAAGTAAGGAC and reverse, CCGAGAAGGTGAGCTTCAGTT; gria4b: forward, ACAA GGCCTGTTGGACAAATTGAAA and reverse, CACGGTAAGT CGGATGACACC; gria $2 b$ : forward, CTGAACGAGCAGGGGCTTC and reverse, AGCATCTGTGAACGTCATCCC; and ef $1 \alpha$ : forward, GAGACTGGTGTCCTCAAGCC and reverse, CCAACGTTGTCA CCAGGAGT. Expression was quantified using ImageJ and compared with relative expression in wild-type larvae.

Statistical analyses

Statistics for all quantitative experiments were conducted using GraphPad Prism. To compare between controls and treatment groups, a two-sided unpaired $t$ test was used. To compare across all genotypes or to compare multiple treatment groups with control, a one-way ANOVA with Tukey's multiple comparison test was used. For quantification of the difference in fluorescence intensity on cell membranes relative to the background, a one-sample $t$ test with a hypothetical value of 1 was used. Categorical data were analyzed using GraphPad Prism's one-tailed Fisher's exact test. The significance level was set at $\alpha=0.05$ ( $p$ s $<0.05$ were considered significant).

\section{Results}

\section{The AMPAR subunit gene gria4a is expressed by spinal cord} OPCs

While the AMPAR subunit genes griala, gria1b, gria $2 b$, and gria $4 a$ are expressed in the developing zebrafish spinal cord, it was unclear whether any were expressed by OPCs (Hoppmann et al., 2008). To determine which, if any, of these genes are selectively expressed in spinal cord OPCs, we performed RNA sequencing of OLCs $\left(\right.$ olig $2^{+} / \operatorname{sox} 10^{+}$cells collected from the trunks of $\operatorname{Tg}$ (olig2:dsred); $\operatorname{Tg}($ sox10:megfp) larvae at $72 \mathrm{hpf}$ ), Schwann cells $\left(\right.$ fox $3^{+} /$sox $10^{+}$cells collected from the trunks of Gt(foxd3:mCherry); $\mathrm{Tg}$ (sox10:megfp) larvae at $72 \mathrm{hpf}$ ), and neurons $\left(n b t^{+}\right.$cells collected from the trunks of $\mathrm{Tg}(\mathrm{nbt}$ :dsred) larvae at $72 \mathrm{hpf}$ ) and compared the expression of each AMPAR subunit gene across these cell types. RNA sequencing revealed that at $72 \mathrm{hpf}$, both gria $2 b$ and gria $4 a$ were highly expressed in OLCs, but gria $4 a$ was the subunit with unique expression in OLCs and was not highly expressed by either Schwann cells or neurons (Fig. 1A). To validate these results, we performed in situ hybridization with a mRNA probe specific to gria $4 a$ at 48 and $72 \mathrm{hpf}$ and found expression in cells closely associated with the spinal cord white matter (Fig. 1B). To confirm that gria $4 a$ was expressed by OLCs, we performed in situ hybridization for gria $4 a$ and immunohistochemistry with an antibody specific to Sox10, which labels OLCs, on $80 \mathrm{hpf} \operatorname{Tg}($ olig2:egfp) larvae, and observed Sox $10^{+} /$olig2 ${ }^{+} /$gria $4 a^{+}$OLCs in the dorsal spinal cord (Fig. 1C). OLCs in the ventral spinal cord were typically Sox $10^{+} /$olig $2^{+} /$gria $4 a^{-}$(Fig. 1C). Together, these results indicate that the AMPAR subunit gene gria4a, which encodes the AMPAR subunit GluR4A, is expressed by a subset of spinal cord OLCs during development.

Because the AMPAR subunit gene gria $4 a$ is highly expressed by OLCs and not neurons at $72 \mathrm{hpf}$ (Fig. 1A), we hypothesized that this gene was a good candidate to mediate OPC migration before myelination. To test this hypothesis, we used CRISPR/ Cas9 gene editing to create mutants that perturbed the GluR4A subunit. We injected a gria $4 a$ gRNA that targeted the sixth exon of the gria4a gene (Fig. 1D), into one-cell embryos and grew these putative mutant founders to adulthood. Upon crossing them to identify germline founders carrying a mutation in gria $4 a$, we identified one allele that caused a 13-bp insertion and subsequent frameshift mutation early in the gene after amino acid 289 (Fig. 1D). This frameshift mutation resulted in a premature stop codon, which terminates the GluR4A polypeptide before translation of the critical glutamate-binding and transmembrane domains (Fig. 1E). These mutant embryos and larvae were morphologically indistinguishable from their clutchmates both at 2 and $5 \mathrm{dpf}$ (Fig. 1F), and adults were homozygous viable.

To investigate whether gria4a expression was reduced in gria $4 a$ mutant embryos and larvae, we performed in situ hybridization and this revealed that there was gria $4 a$ transcript in the spinal cord of gria $4 a^{\text {uva } 43 / \text { uva } 43}$ mutants, both at 48 and $72 \mathrm{hpf}$, although at lower levels than seen in wild-type controls (compare Fig. $1 G$ and $B$ ). To confirm this reduction in transcript level, we performed RT-PCR and observed that in $48 \mathrm{hpf}$ gria $4 a^{\text {uva } 43 / \text { uva } 43}$ embryos, gria $4 a$ mRNA expression was decreased by $\sim 20 \%$ when compared with wild-type larvae (mutant expression of $81.1 \pm 1.3 \%$ of wild-type level; Fig. $1 H$ ), possibly caused by nonsense-mediated decay. Because loss of gria 4 a may result in genetic composition by other AMPAR subunits and an altered composition of subunits may change $\mathrm{Ca}^{2+}$ influx into the cells, we also analyzed expression of gria $4 b$ and gria $2 b$. We observed that while expression of gria $4 b$ was low in wild-type larvae, expression increased both in heterozygous (146.1 $\pm 34.7 \%$ increase compared with the wild-type level) and homozygous larvae $(142.0 \pm 36.5 \%$ increase compared with the wild-type level). Additionally, gria2 $b$ expression also increased in heterozygous (123.6 $\pm 14.5 \%$ increase compared with the wild-type level) and homozygous larvae $(124.8 \pm 17.8 \%$ increase compared with the wild-type level; Fig. $1 H)$. Thus, in gria $4 a^{\text {uva } 43 / u v a 43}$ and gria $4 a^{+/ u v a 43}$ larvae there are reduced levels of GluR4A, as well as increased expression of GluR4B and GluR2B, indicating there may be some compensation for the loss of a functional copy of the gria $4 a$ gene.

\section{GluR4A is required for OPC migration and distribution in the spinal cord}

We hypothesized that mutation of GluR4A would likely affect OPC sensing of the neurotransmitter glutamate as we recently identified neuronal activity as important for OPC migration (Fontenas et al., 2019). Previous studies demonstrate that glutamate can be released at sites along CNS axons outside of the synapse (Kukley et al., 2007; Ziskin et al., 2007; Wake et al., 2015; Kula et al., 2019). Therefore, we hypothesized that gria $4 a^{\text {uva } 43 / \text { uva } 43}$ mutants would exhibit abnormal OPC migration because they would be unable to sense this axonallyderived glutamate. To investigate this hypothesis, we performed 
A

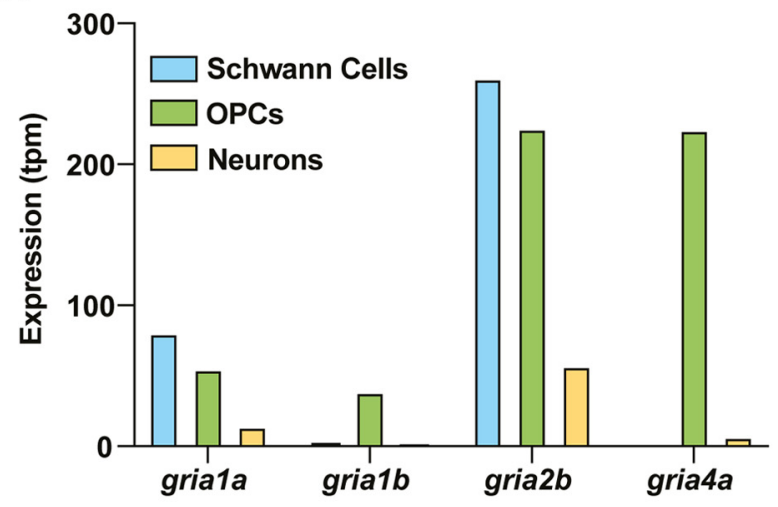

B
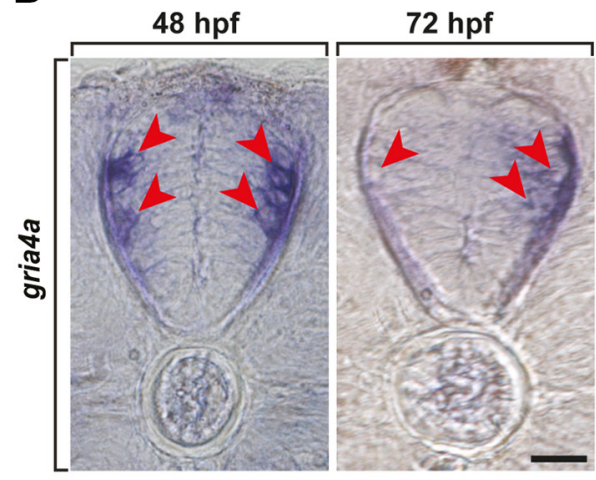

C
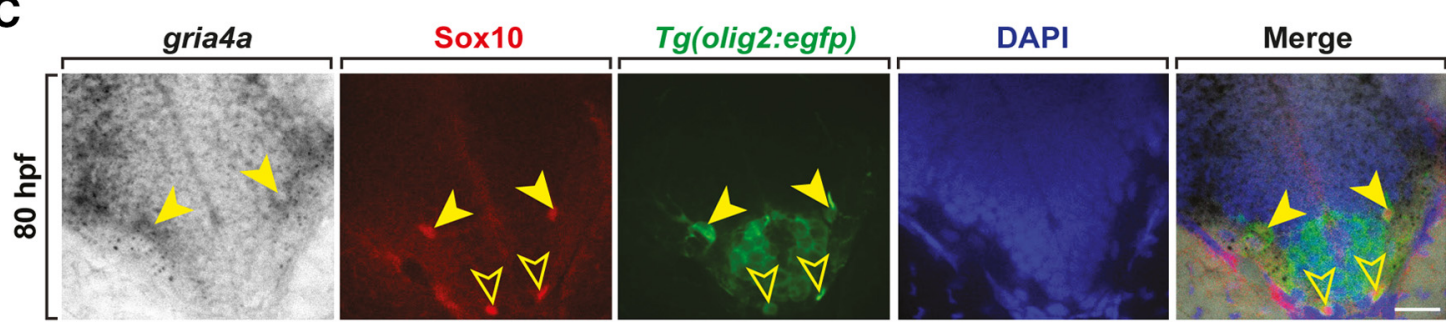

D

gria4a: CTT GAG AGG TTC ATG CAC GGC GGG GCG AAC GTA ACT GGT TTC CAG TTG GTG GAT TTC AGC AAA CCG ATG GTG ATC AAA CTA ATG CAG CGC TGG AAC AAA CTA GAC CAG AGG GAG TAT CCG GGA TCT GAA...

$\mathrm{Glu}^{286}$ > Stop: CTT GAG AGG TTC ATG CAC GTT CAT GCA CGT TCG CGG GGC GAA CGT AAC TGG TTT CCA GTT GGT GGA TTT CAG CAA ACC GAT GGT GAT CAA ACT AAT GCA GCG CTG GAA CAA ACT AGA CCA GAG GGA GTA TCC GGG ATC TGA

E gRNA sequence $\quad$ PAM site $\quad$ 13bp insertion Stop codon

GluR4A wildtype polypeptide: 898 AAs

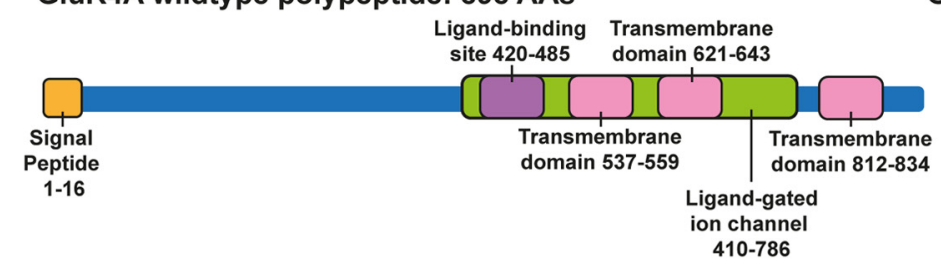

GluR4A mutant polypeptide: 289 AAs

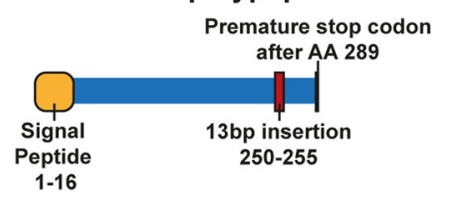

$\mathbf{F}$

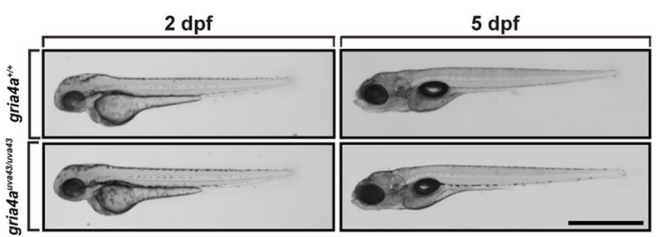

G

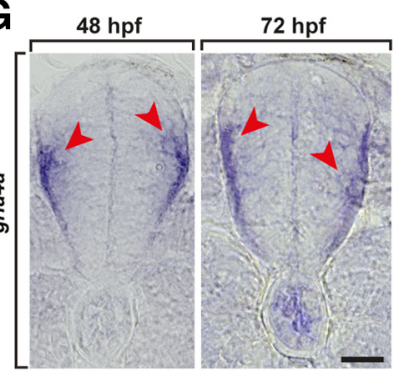

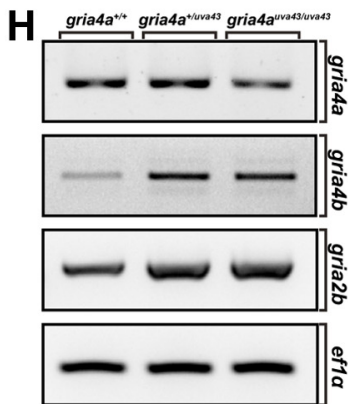

Figure 1. The AMPAR subunit gene gria4a is expressed in spinal cord OPCs. $\boldsymbol{A}$, Expression of AMPAR subunits by cell type, in transcripts per million (tpm). $\boldsymbol{B}$, In situ hybridization shows gria4a expression (red arrows) in wild-type spinal cord cross-sections at 48 and $72 \mathrm{hpf}$ (scale bar: $20 \mu \mathrm{m}$ ). C, In situ hybridization for gria4a mRNA, immunohistochemistry for Sox10, and trans-

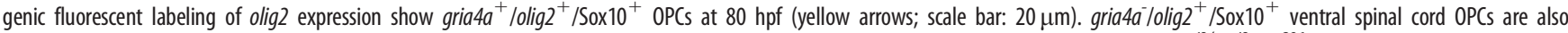

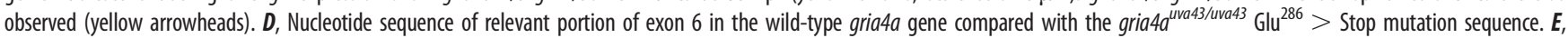

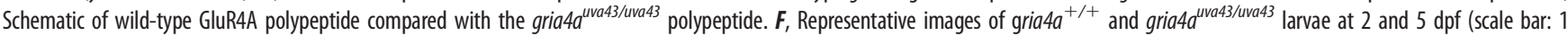

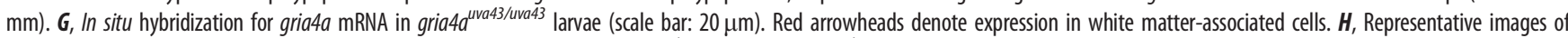
RT-PCR of gria4a, gria4b, gria $2 b$, and ef1 $\alpha$ mRNA at 48 hpf in wild-type, gria4a ${ }^{+/ \text {/ua } 43}$, and gria $4 a^{\text {uva } 43 / \text { /uva } 43}$ embryos. Ef1 $\alpha$ was used as housekeeping gene.

in vivo, time-lapse imaging using $\operatorname{Tg}$ (olig2:dsred); $\operatorname{Tg}(\mathrm{mbp}$ :egfp$C A A X)$ transgenic embryos and larvae, where olig2 and $m b p$ regulatory sequences drive expression of fluorescent reporters in OPCs and motor neurons for olig2, and in myelin-producing cells for $m b p$, and imaged the dorsal migration of spinal cord OPCs (Shin et al., 2003; Almeida et al., 2011). In time-lapse movies from 56 to $72 \mathrm{hpf}$, we observed similar numbers of dorsal OPCs across genotypes at $56 \mathrm{hpf}$, which is just as these cells are migrating from their ventral spinal cord origin (Fig. 2A). However, by $72 \mathrm{hpf}$, there were significantly fewer dorsal OPCs in gria $4 a^{\text {uva } 43 / \text { uva } 43}$ larvae compared with wild-type clutchmates $(p=0.0041$; Fig. $2 B)$ and a similar trend was observed compared 
A

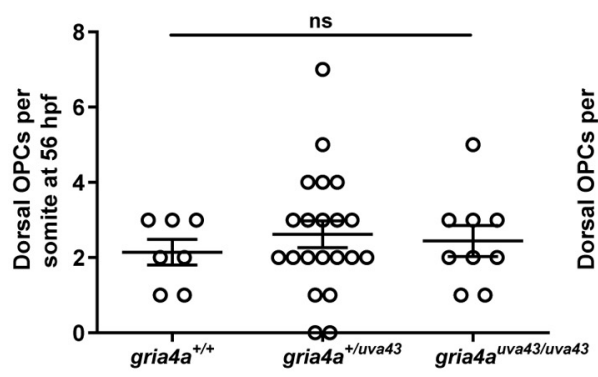

B

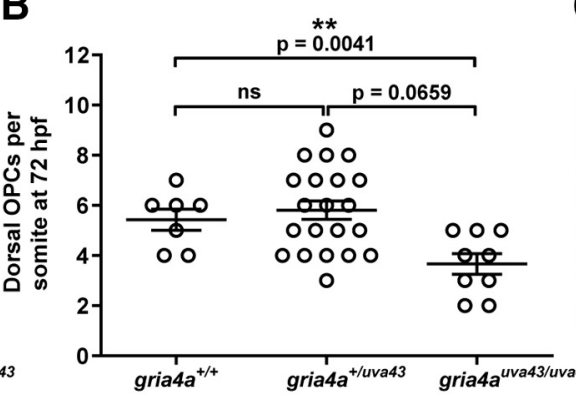

C

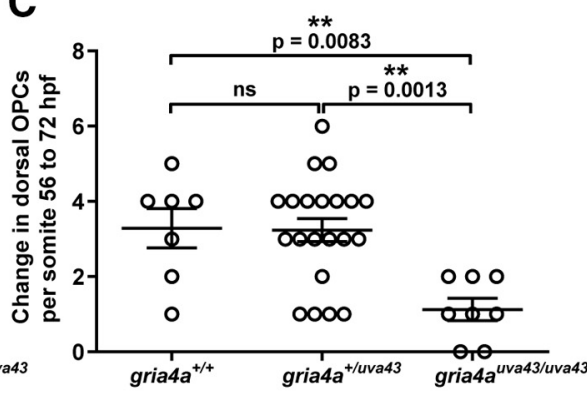

D

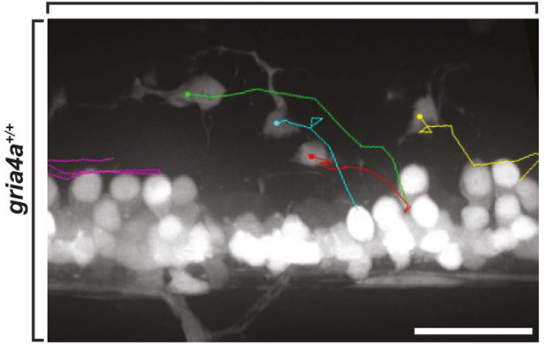

E

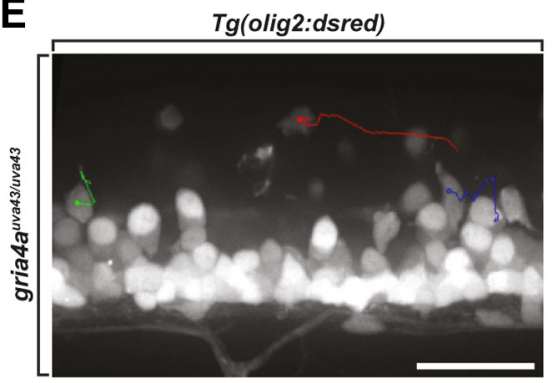

H

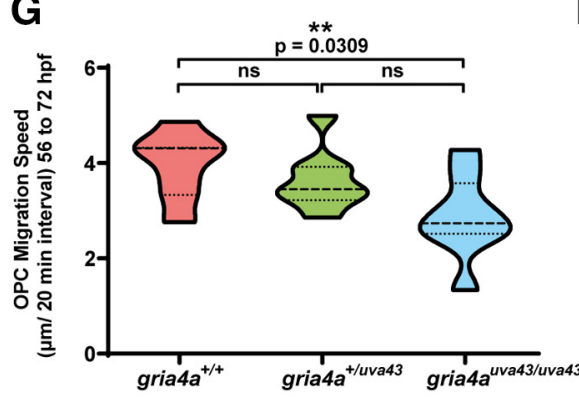

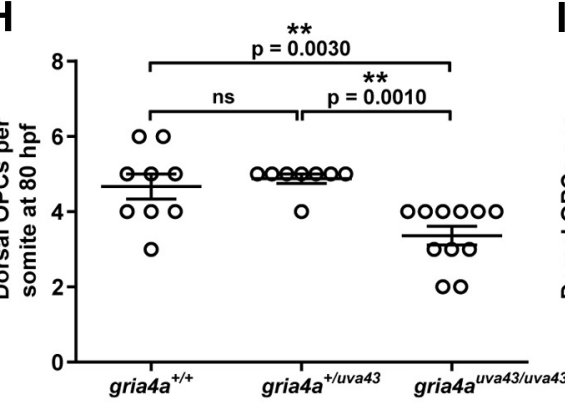

$\mathbf{F}$

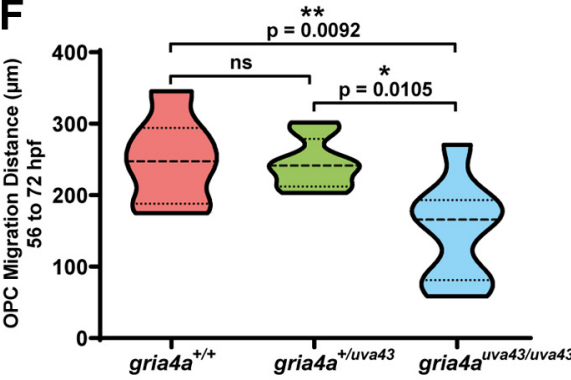

I

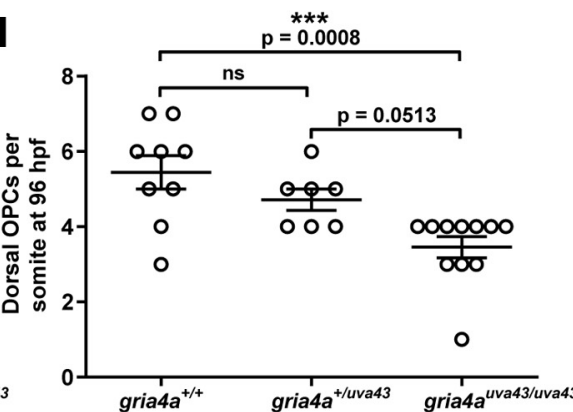

$\mathbf{J}$

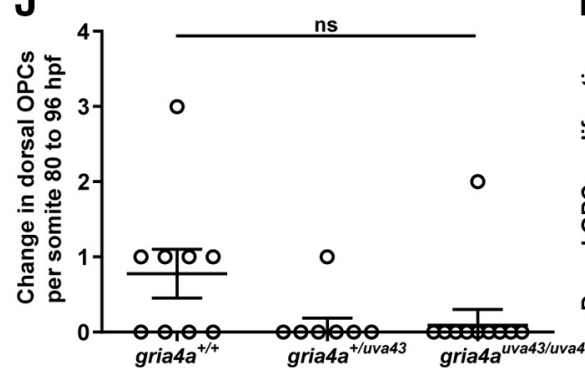

K

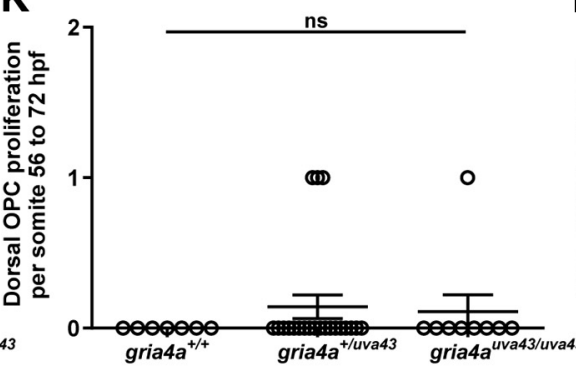

$\mathbf{L}$

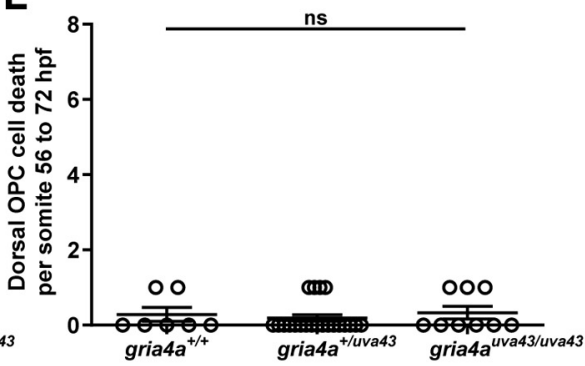

Figure 2. Gria4a mutants have reduced dorsal OPC migration and altered OPC migration dynamics. A, B, Mean \pm SEM of dorsal OPCs per somite in Tg(olig2:dsred); $T g(m b p: e g f p-(A A X)$

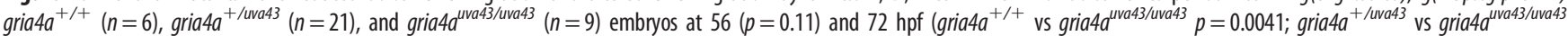

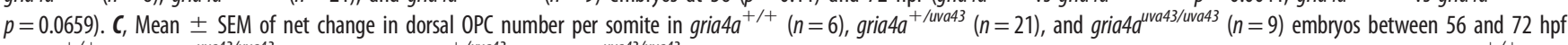

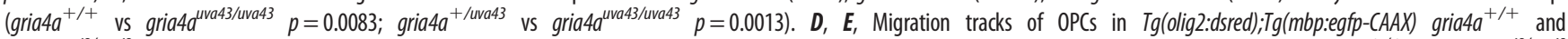
gria4ava43/uva43 embryos between 56 and 72 hpf. Only fluorescence from $T g$ (olig2:dsred) line shown for clarity. $\mathbf{F}, \mathbf{G}$, Violin plots of OPC migration distance (gria4a ${ }^{+/+}$vs gria4ava43/uva43

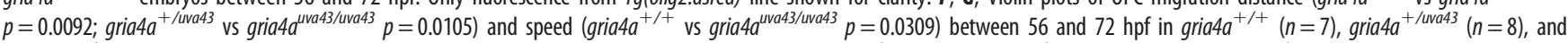

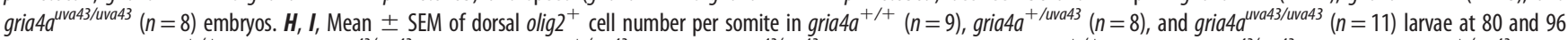

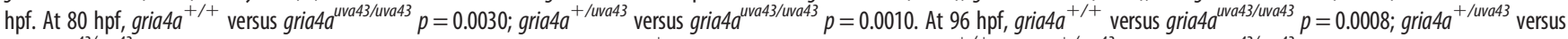
gria4a $a^{\text {uva43/uva43 }} p=0.00,513$. J, Mean \pm SEM of net change in dorsal olig2 ${ }^{+}$cell number per somite in gria4a $a^{+/+}$, gria4a ${ }^{+/ 4 v a 43}$, and gria4ava43/uva43 larvae between 80 and 96 hpf; $p=0.0794$ across all groups. $\boldsymbol{K}, \boldsymbol{L}$, Quantification of dorsal OPC proliferation $(\boldsymbol{K})$ and cell death $(\boldsymbol{L})$ from in vivo, time-lapse movies of wild-type $(n=7)$, heterozygous $(n=21)$, and mutant $(n=9)$ larvae between 56 and $72 \mathrm{hpf}$. ns $=$ no significant differences between groups. Scale bar: $50 \mu \mathrm{m}$.

with heterozygous clutchmates ( $p=0.0659$; Fig. $2 B$ ). When we compared the difference between dorsal OPCs at $72 \mathrm{hpf}$ and 56 hpf, OPCs in gria4a ${ }^{\text {uva } 43 / \text { vva } 3}$ larvae exhibited a significantly reduced net dorsal migration when compared with wild-type $(p=0.0083)$ and heterozygous $(p=0.0013)$ larvae (Fig. $2 C)$. These results demonstrate that gria $4 a$ is necessary for dorsal OPC migration by $72 \mathrm{hpf}$.
To better understand what causes the reduced dorsal migration, we analyzed our time-lapse videos using the Manual Tracking ImageJ plugin for cell tracking (Fig. 2D-G). Quantification of distance migrated revealed that OPCs in gria4a $a^{\text {uva } 43 / \text { uva } 43}$ larvae migrated a shorter distance $(p=0.0092$; Fig. $2 F)$ and at a slower speed ( $p=0.0309$; Fig. $2 G$ ) than their wild-type counterparts between 56 and $72 \mathrm{hpf}$. Interestingly, 
A

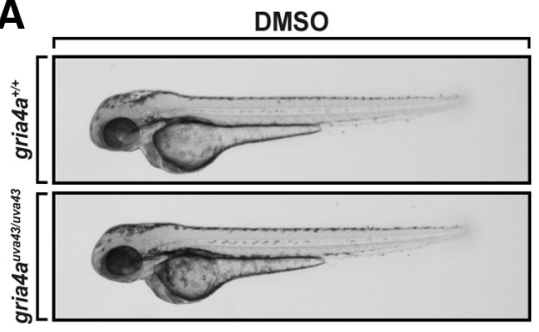

NBQX

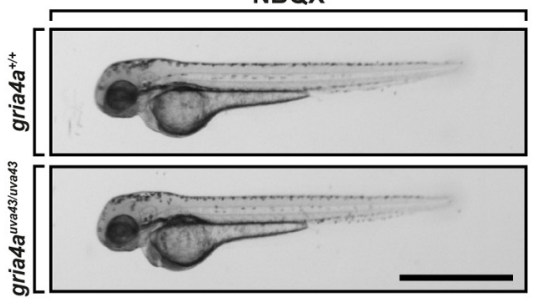

gria4a $a^{+/+}$

C

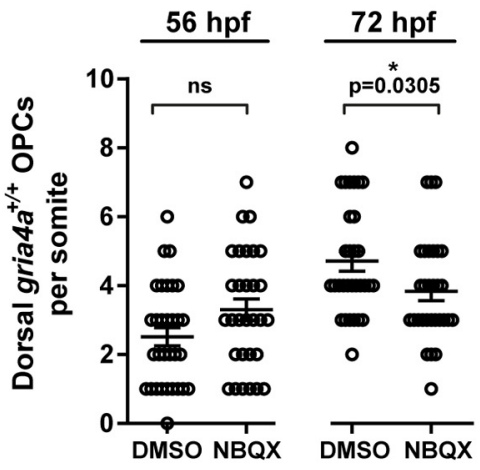

B
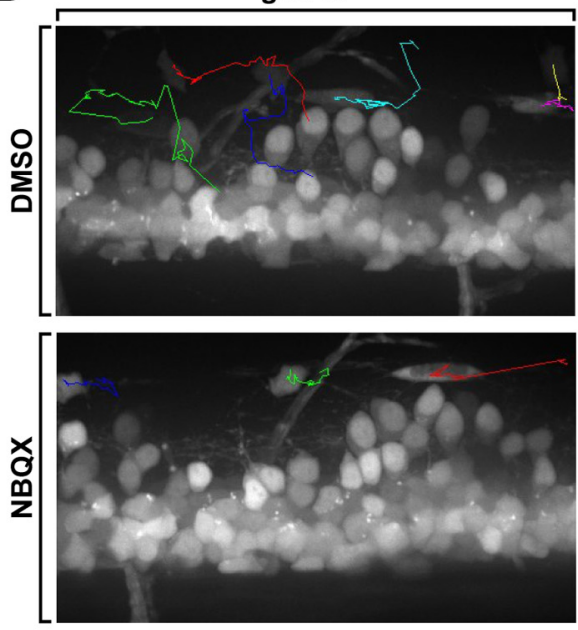

gria4a $a^{+/ u v a 43}$

D

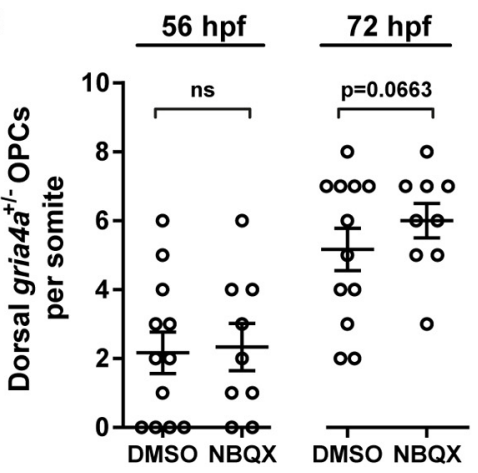

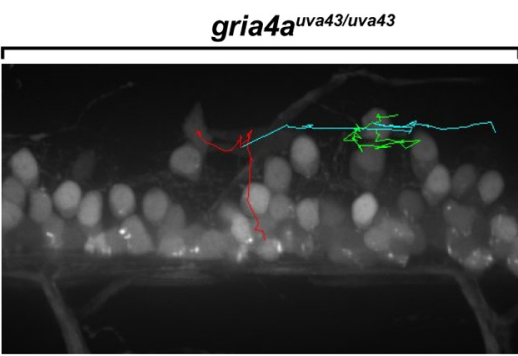

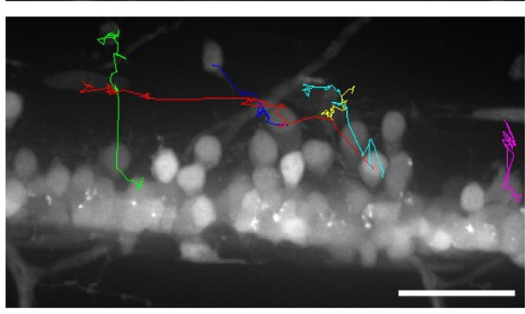

gria4a $a^{u v a 43 / u v a 43}$

E

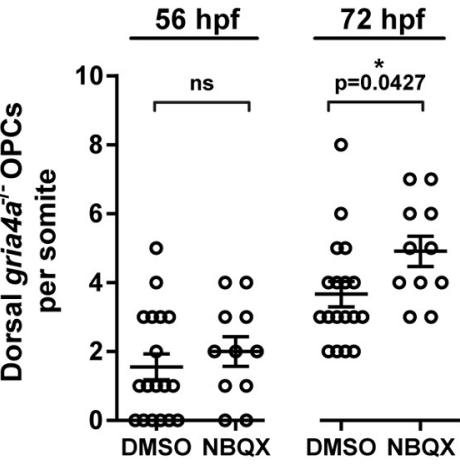

Figure 3. Treatment with ionotropic glutamate receptor inhibitor NBQX reduces dorsal OPC migration between 56 and $72 \mathrm{hpf}$. A, Brightfield images of 56-hpf gria4a wild-type and mutant embryos after treatment with DMSO or $40 \mu \mathrm{m}$ NBQX. $\boldsymbol{B}$, Representative images and tracing of OPC migration after treatment with $40 \mu \mathrm{m}$ NBQX (scale bar: $50 \mu \mathrm{m}$ ). C-E, Mean \pm SEM of dorsal OPCs per somite at 56 to $72 \mathrm{hpf}$ in $\mathrm{Tg}(\mathrm{nkX2.2a:megfp);Tg(olig2:dsred)} \mathrm{embryos} \mathrm{treated} \mathrm{with} 40 \mu \mathrm{M}$ NBQX in 1\% DMS0 or 1\% DMSO alone from 30 to 72 hpf. C, Quantification of dorsal OPCs per somite in DMSO and NBQX-treated gria4a ${ }^{+/+}$embryos and larvae at 56 and $72 \mathrm{hpf}$ (DMSO $n=31$, NBQX $n=30$, DMSO vs NBQX at $72 \mathrm{hpf} p=0.0305$ ). D, Quantification of dorsal OPCs per somite in DMSO and NBQX-treated gria4a $a^{+/ \text {iva } 43}$ embryos and larvae at 56 and 72 hpf (DMSO $n=12, \operatorname{NBQX} n=11$, DMSO vs NBQX at 72 hpf $p=0.0663$ ). E, Quantification of dorsal

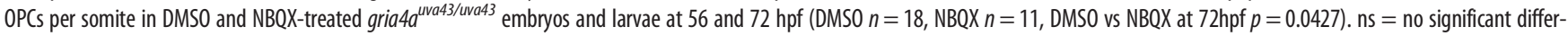
ences between groups.

although gria $4 a^{+/ u v a 43}$ OPCs migrated a significantly longer distance than gria $4 a^{\text {uva } 43 / u v a 43}$ OPCs $(p=0.0105$; Fig. $2 F)$, their migration speed, although not significantly different from either wild-type or mutant OPCs, was intermediate between the two (Fig. $2 G$ ). To determine whether the difference in migration between wild-type and mutant OPCs was the result of a developmental delay, we repeated the experiment at a later stage and imaged the larvae from 80 to $96 \mathrm{hpf}$. The migration during this period revealed that there were fewer dorsal olig $2^{+}$ cells in gria $4 a^{\text {uva } 43 / u v a 43}$ larvae compared with gria $4 a^{+/+}$and gria $4 a^{+ \text {uva } 43}$ larvae at both $80\left(p=0.0030\right.$ gria $4 a^{\text {uva } 43 / \text { uva } 43}$ vs

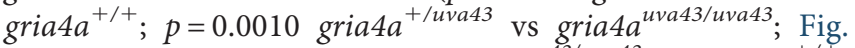
$2 H)$ and $96 \mathrm{hpf}\left(p=0.0008\right.$ gria $4 a^{\text {uva } 43 / \text { uva } 43}$ vs $\operatorname{gria}^{4} a^{+/+}$; $p=0.0513$ gria $4 a^{+/ \text {uva } 43}$ vs gria4a $a^{\text {uva } 43 / \text { uva } 43} ;$ Fig. 2I). Additionally, there was minimal net dorsal OLC migration with no significant difference across the genotypes from 80 to $96 \mathrm{hpf}$ (Fig. 2J), which demonstrates that the variation in OLC migration observed from 56 to $72 \mathrm{hpf}$ was not the result of a developmental delay. Finally, we wanted to determine whether there were any changes to OPC proliferation or survival in gria4a mutant larvae, which has previously been described (Kougioumtzidou et al., 2017). To do this, we quantified the number of cell divisions and cell death events from in vivo, time-lapse imaging data taken from 56 to $72 \mathrm{hpf}$. These studies revealed that OPC proliferation and survival were unchanged in the gria $4 a^{\text {uva } 3 / \text { uva } 43}$ larvae when compared with heterozygous and wild-type siblings (Fig. $2 K, L$ ). Together, these findings demonstrate that mutation of GluR4A results in reduced dorsal OPC migration and altered migration dynamics of spinal cord OPCs from 56 to $72 \mathrm{hpf}$.

To independently confirm that perturbed AMPAR signaling was the cause of the dorsal OPC migration defect we observed in gria $4 a^{\text {uva } 3 / \text { uva } 43}$ mutants, we used the drug NBQX, an ionotropic glutamate receptor inhibitor (Deng et al., 2003; Evonuk et al., $2020)$ to investigate the role of AMPAR signaling in OPC migration. To do this, we treated $\operatorname{Tg}($ olig2: $d s r e d) ; \operatorname{Tg}(n k x 2.2 a$ :megfp) embryos from 30 to $72 \mathrm{hpf}$ with either $40 \mu \mathrm{M}$ NBQX dissolved in $1 \%$ DMSO or with $1 \%$ DMSO as a control. We then performed in vivo, time-lapse imaging from 56 to $72 \mathrm{hpf}$ and compared dorsal OPC migration between the two groups. Similar to gria $4 a$ mutant embryos and larvae, wild-type embryos treated with NBQX and DMSO larvae looked healthy (Fig. 3A). When we assayed OPC migration between 56 and $72 \mathrm{hpf}$, we observed a significant reduction in the number of $n k \times 2.2 a^{+} /$olig $2^{+}$OPCs that migrated dorsally between 56 and $72 \mathrm{hpf}$ in NBQX-treated larvae compared with DMSO-treated larvae $(p=0.0305$; Fig. $3 B$, 
C). Similar to what we observed in gria $4 a$ mutant larvae, OPC proliferation and cell death were not affected in wild-type larvae treated with NBQX (proliferation: DMSO $0.40 \pm 0.51$ cells per somite vs NBQX $0.38 \pm 0.51$ cells per somite; cell death: DMSO $0.20 \pm 0.41$ cells per somite vs NBQX $0.46 \pm 0.66$ cells per somite; data not shown).

To reveal any compensatory mechanisms that may be taking place in gria $4 a$ mutant larvae, we treated both gria $4 a^{+/ u v a 43}$ and gria $4 a^{\text {uva } 43 / \text { uva } 43}$ embryos with NBQX from 30 to $72 \mathrm{hpf}$, and imaged OPC migration from 56 to $72 \mathrm{hpf}$. As OPCs already migrate significantly less in mutants, we did not expect to observe any effect of NBQX on dorsal OPC migration in mutant larvae. However, to our surprise, we observed a trend of increased dorsally-migrated OPCs at $72 \mathrm{hpf}$ in NBQX-treated gria4a heterozygous larvae ( $p=0.0663$; Fig. $3 D)$, and a significant increase in dorsal OPCs at $72 \mathrm{hpf}$ in gria $4 a$ mutant larvae when compared with DMSO-treated mutant larvae $(p=0.0427$; Fig. $3 B, E)$. We hypothesize that this observed difference in the effect of NBQX on migration between wild-type and mutant larvae may be related to the altered composition of the AMPAR subunits in gria $4 a$ mutant larvae, thereby changing the calcium permeability of the AMPARs, and we will discuss this further in the discussion. Similar to what we observed in wild-type larvae, OPC proliferation and cell death were unchanged in heterozygous and mutant larvae treated with NBQX (gria $4 a^{+/ u v a 43}$ proliferation: DMSO $0.50 \pm 0.90$ cells per somite vs NBQX $0.18 \pm 0.40$ cells per somite; cell death: DMSO $0.17 \pm 0.39$ cells per somite vs NBQX $0.55 \pm 0.69$ cells per somite; gria $4 a^{\text {uva } 43 / \text { uva } 43}$ proliferation: DMSO $0.18 \pm 0.40$ cells per somite vs NBQX $0.25 \pm 0.25$ cells per somite; cell death: DMSO $0.27 \pm 0.47$ cells per somite vs NBQX $0.17 \pm 0.39$ cells per somite; data not shown). Based on the effects of NBQX on wild-type and mutant OPC migration (Fig. $3 C, E$ ), we conclude that AMPAR signaling plays a key role in promoting dorsal OPC migration, and this finding provides validation for the OPC migration defects observed in gria $4 a$ mutants.

To confirm that the reduction in OPC migration was caused by an inability of the gria $4 a^{u v a 43 / u v a 43}$ OPCs to detect glutamate, we locally increased the availability of glutamate by photo-uncaging MNI-glutamate in the dorsal the spinal cord at $56 \mathrm{hpf}$ (Hughes and Appel, 2020). To verify that the glutamate-uncaging method we used was functional, we used a fluorescent glutamate biosensor (SF-iGluSnFR; Marvin et al., 2018), which is a cell surface biosensor that detects extracellular glutamate, expressed under the sox10 promoter. To do this, we incubated $\mathrm{Tg}$ (sox10:SF-iGluSnFR) larvae with $1 \mu \mathrm{M}$ MNI-glutamate in $0.4 \%$ DMSO for $1 \mathrm{~h}$ before imaging. We then mounted the larvae for in vivo imaging and focally uncaged the MNI-glutamate using a 404-nm pulsed nitrogen dye laser in a region of interest just dorsal to iGluSnFR ${ }^{+}$cells $(\sim 20-40 \mu \mathrm{m}$ from measured regions on OPC membranes, in line with previous studies used for uncaging MNI-glutamate; Fig. 4A, red circle; Li et al., 2012; Hughes and Appel, 2020). We then imaged every $32 \mathrm{~ms}$ for $1 \mathrm{~min}$ before and immediately after uncaging of the MNI-glutamate and compared the t-stacks of the two time-lapses. We then measured the change in fluorescence intensity across SF-iGluSnFR ${ }^{+}$ OPC membranes and size-matched control regions immediately next to iGluSnFR ${ }^{+}$cells (Fig. 4A-C, yellow regions mark cell membranes and cyan regions mark neighboring background). After uncaging, we observed larger changes in iGluSnFR fluorescence intensity over time on sox $10^{+}$cell membranes compared with size-matched regions in the background (Fig. 4B, representative data). Quantification of the change in fluorescence of the t-stacks revealed that the uncaging of MNI-glutamate resulted in significant changes in SFiGluSnFR fluorescence on the cell-membranes of sox $10^{+}$cells compared with size-matched regions of background $(p<$ 0.0001; Fig. 4C). From these results, we conclude that OPCs can detect glutamate release in the spinal cord.

Once we were confident that OPCs can respond to glutamate in vivo, we performed in vivo, time-lapse imaging from 56 to 58 hpf in $\mathrm{Tg}$ (olig2:dsred);gria4 ${ }^{+/+}$, gria $4 a^{+/ \text {uva } 43}$, and gria $4 a^{\text {uva } 43 /}$ ${ }^{\text {uva } 43}$ larvae after focal uncaging of MNI-glutamate in the dorsal spinal cord (uncaging occurred $\sim 20-40 \mu \mathrm{m}$ from SF-iGluSnFR ${ }^{+}$ OPCs; Fig. 4C). After uncaging, we observed that ${\text { gria } 4 a^{+/+}}^{+}$ OPCs increased their migration speed $(p=0.0048$; Fig. $4 D)$ and average migration distance $(p=0.0094$; Fig. $4 E)$ in response to the increased local availability of glutamate. In contrast, both gria $4 a^{+/ \text {uva } 43}(p=0.0018)$ and gria4a $a^{u v a 43 / u v a 43}$ OPCs $(p<$ 0.0001 ) migrated significantly slower (Fig. $4 D$ ) and for shorter distances (gria4a $a^{+/ \text {uva } 43} \quad p=0.0012 ;$ gria4a $a^{\text {uva } 3 / \text { uva } 43}$ OPCs $p<0.0001)$ when compared with $g r i a 4 a^{+/+}$OPCs in response to the local glutamate burst. In fact, gria $4 a^{\text {uva } 43 / u v a 43}$ OPCs treated with DMSO were indistinguishable from gria $4 a^{\text {uva } 43 / \text { uva } 43}$ OPCs exposed to uncaged MNI-glutamate, demonstrating that mutation of gria4a affects OPC sensing of, and migration toward, glutamate.

Next, we sought to determine whether the altered OPC migration we observed in gria $4 a^{u v a 43 / u v a 43}$ larvae was maintained past developmental myelination stages, at $6 \mathrm{dpf}$. To investigate this, we performed immunohistochemistry on transverse trunk sections of mutant and control larvae to investigate the number and distribution of Sox $10^{+}$spinal cord OPCs and oligodendrocytes at 3 and $6 \mathrm{dpf}$. For each spinal cord section, we counted and recorded the XY-coordinates of each Sox $10^{+}$cell, then compiled these coordinates to generate heat maps for each developmental stage and genotype (Fig. 5A,B). We stratified the positional data into dorsal (upper $2 / 3$ of $Y$ area) and ventral (lower $1 / 3$ of $\mathrm{Y}$ area) categories, roughly divided by the upper boundary of the primary motor neuron ( $\mathrm{pMN}$ ) domain. Based on these categories, we quantified dorsal and ventral Sox $10^{+}$cells in each genotype. At both stages, there were significantly fewer dorsal Sox $10^{+}$cells in the gria4a $a^{u v a 43 / u v a 43}$ spinal cord compared with the $g r i a 4 a^{+/+}$spinal cord, and there were more ventral Sox $10^{+}$cells in the gria $4 a^{\text {uva } 43 / \text { vva } 43}$ spinal cord compared with the gria $4 a^{+/+}$spinal cord at both $3(p=0.0080$; Fig. $5 A, C)$ and 6 $\operatorname{dpf}(p=0.0159$; Fig. $5 B, E)$, based on the proportion of dorsal cells in each section $(n=20$ for all groups at $3 \mathrm{dpf}, \mathrm{n}=10$ for all groups at $6 \mathrm{dpf}$ ). Interestingly, at $3 \mathrm{dpf}$, there were slightly more dorsal OPCs in gria $4 a^{+/ \text {uva43 }}$ larvae compared with in gria $4 a^{+/+}$larvae $(p=0.0478$; Fig. $5 A, C)$. However, at both timepoints, the total number of Sox $10^{+}$cells (both dorsal and ventral) across genotypes was similar (Fig. $5 F$ ), which demonstrates that cell proliferation and survival were not affected by the gria $4 a$ mutation, and this matches the proliferation and cell death counts we did previously (Fig. $2 K, L$ ). These results reveal that the OPC migration defect in gria $4 a^{u v a 43 / u v a 43}$ larvae persists beyond early development and that GluR4A is necessary for normal distribution of OPCs throughout the spinal cord.

\section{Mutation of gria4a alters OPC migration in a cell- autonomous manner}

Our gria4a CRIPSR mutant line is a global mutant, so we sought to determine whether the observed OPC migration differences were cell-autonomous, or alternatively, the consequence of aberrant GluR4A in other cell types, including neurons. To 


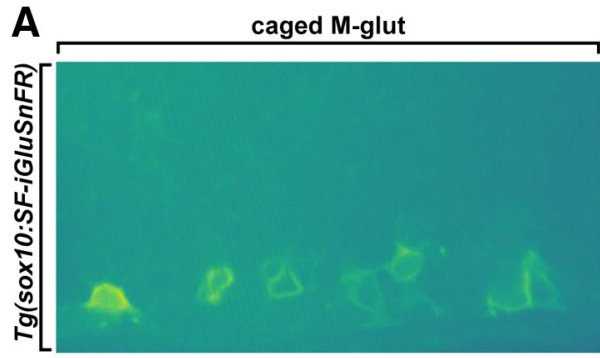

B

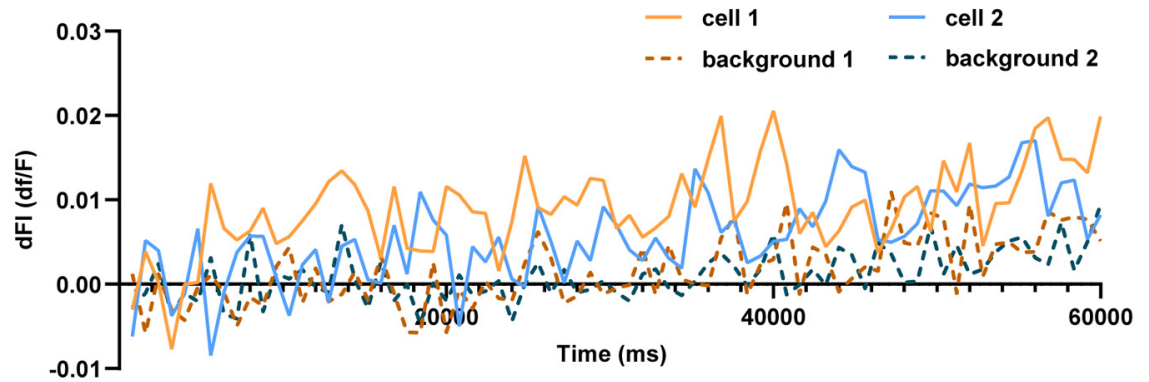

D

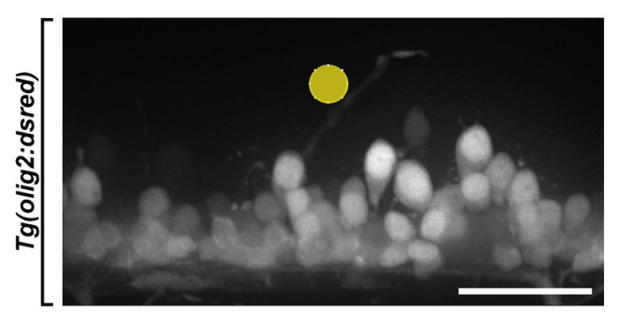

uncaged M-glut

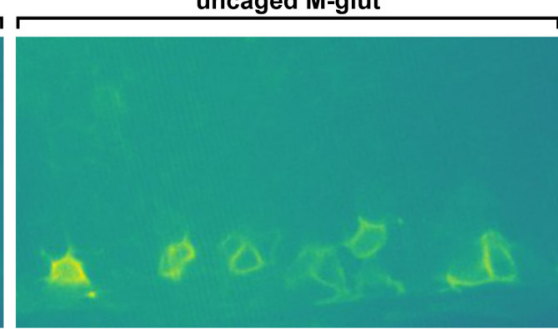

E

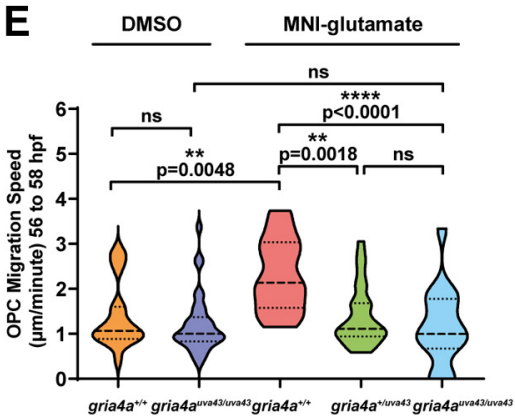

selected regions

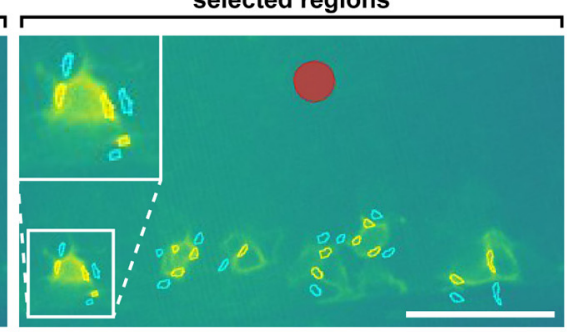

C

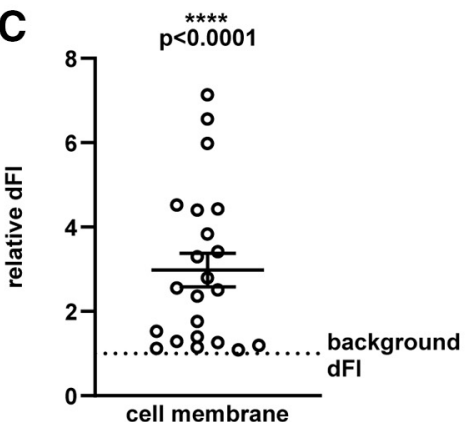

$\mathbf{F}$

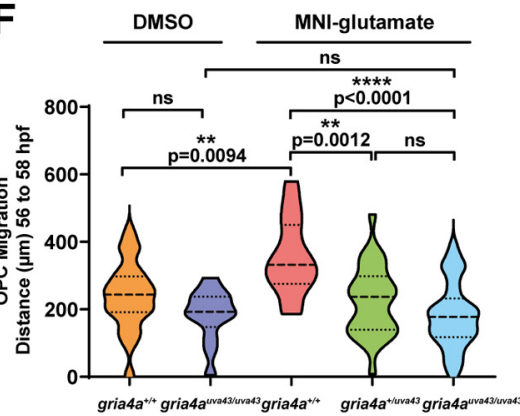

Figure 4. gria4a mutant OPCs have a decreased ability to sense glutamate. A, Representative images of $56 \mathrm{hpf} \mathrm{Tg}(\mathrm{sox} 10$ :SF-iGluSnFr) embryos before and after photo-uncaging of MNI-glutamate (M-glut) by a 404-nm laser. Red circle indicates focal region of interest (ROl) where M-glut was uncaged, $\sim 20-40 \mu \mathrm{m}$ from sox $10^{+}$OPC membranes. Yellow ROls indicate where fluorescence intensity was measured on OPC membranes and cyan ROls indicate size-matched regions where background fluorescence was measured next to the cell ( $n=22$ regions of $n=18$ cells in $n=3$ fish). Inset is magnified view of a SF-iGluSnFR ${ }^{+}$cell fluorescence after M-glut uncaging. $\boldsymbol{B}$, Representative quantification of the difference in fluorescence intensity (dFI) before and after MNI-glut uncaging of two different sox $10^{+}$cell membranes and size-matched ROls in the background, averaged per 25 frames ( $800 \mathrm{~ms}$ ) of imaging. C, Quantification of relative total dFI after uncaging of M-glut on sox $10^{+}$cell membranes compared with the size-matched regions in the background $(n=22, p<0.0001)$. D. Representative image of ROl (yellow, $\left.50 \mu \mathrm{m}^{2}\right)$ in the spinal cord where M-glut was uncaged in Tg(oligz:dsred) embryos at $56 \mathrm{hpf}$ for data collected in $\boldsymbol{D}, \boldsymbol{E}$. $\boldsymbol{E}, \boldsymbol{F}$, Violin plots of OPC migration speed and distance in gria4a $a^{+/+}$(DMSO $n=9, \mathrm{M}-\mathrm{glut}$ $n=6$ ), gria4a ${ }^{+/ \text {/uva43 }}$ (M-glut $n=12$ ), and gria4a ava43/vva43 (DMSO $n=6$, M-glut $n=15$ ) larvae after uncaging of M-glut $(1 \mu \mathrm{M})$ over a time course of $2 \mathrm{~h}$. Migration speed across all groups

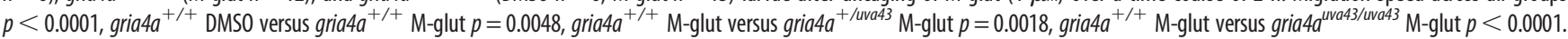
Migration distance across all groups $p<0.0001$, gria4a $a^{+/+}$DMSO versus gria4a ${ }^{+/+} \mathrm{M}$-glut $p=0.0094$, gria $4 a^{+/+}$M-glut versus gria $4 a^{+/ \text {uva43 }} \mathrm{M}$-glut $p=0.0012$, gria4a $a^{+/+} \mathrm{M}$-glut versus

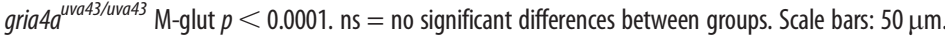

investigate this, we created genetic mosaics (Gansner et al., 2017). To do so, we transplanted blastula cells from dextraninjected gria4a $a^{\text {uva43/uva43 }} ; \operatorname{Tg}(m b p: e g f p-C A A X)$ embryos into gria $a^{+/+}$embryos of the same stage, then used in vivo, timelapse imaging to track the migration and dorsal/ventral location of transplanted cells from 56 to $72 \mathrm{hpf}$ (Fig. 6A). At $56 \mathrm{hpf}$,

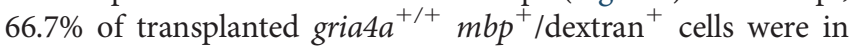
the dorsal spinal cord, whereas only $37 \%$ of transplanted gria4a $a^{\text {uva43/uva43 }} \mathrm{mbp}^{+} /$dextran $^{+}$cells were located dorsally $(p=0.0417$; Fig. $7 B)$. At $72 \mathrm{hpf}$, this difference was even more pronounced, and we observed $83.3 \%$ of the transplanted ${\text { rria } 4 a^{+/+}} m b p^{+} /$dextran $^{+}$cells in the dorsal spinal cord, compared with $28 \%$ dorsally-located, transplanted gria4a $a^{\text {uva } 43 / \text { uva } 43} \mathrm{mbp}^{+} /$dextran ${ }^{+}$cells $(p=0.0003$; Fig. $6 C)$.

To further characterize the cell-autonomy of gria $4 a^{\text {uva } 43 / \text { uva } 3}$ in OLCs, we analyzed the migration dynamics of the transplanted cells and found that gria4a $a^{\text {uva43/uva43 }}$ cells transplanted into gria $4 a^{+/+}$host embryos migrated at a slower speed $(p<0.0001$; Fig. $6 D)$ and for a shorter total distance $(p<0.0001$; Fig. $6 E)$ from 56 to 72 hpf, as compared with transplanted gria $4 a^{+/+}$cells, similar to what we observed in gria $4 a^{\text {uva43/uva43 }}$ larvae. Interestingly, we observed the same trend with $g r i a 4 a^{+/ u v a 43}$ OLCs with respect to a slower migration speed, although it was not significantly different from wild-type OLCs $(p=0.0783$; Fig. $6 D)$. However, gria $4 a^{+/ \text {uva } 43}$ OLCs did migrate significantly less distance than OLCs in ${\text { gria } 4 a^{+/+}}$larvae $(p=0.0011$; Fig. $6 E$ ), which we hypothesize is

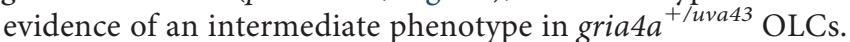
Combined, these results indicate that the spinal cord OPC migration and regional distribution phenotypes we observed in gria4a $a^{\text {uva } 43 / \text { uva }}$ embryos and larvae are cell-autonomous, and mutation of gria4a solely in OPCs is sufficient to disrupt their migration.

To determine whether there were any non-cell-autonomous effects of gria $4 a$ on OLC migration, we transplanted blastula cells from dextran-injected $g r i a 4 a^{+/+} ; \operatorname{Tg}(m b p: e g f p-C A A X)$ embryos 
A

$3 \mathrm{dpf}$

B

$6 \mathrm{dpf}$
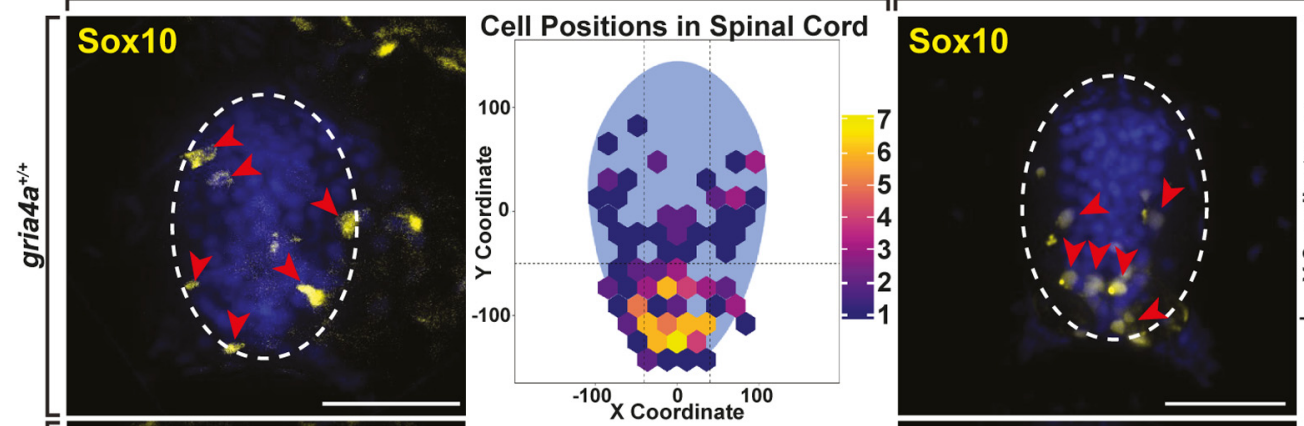

Cell Positions in Spinal Cord
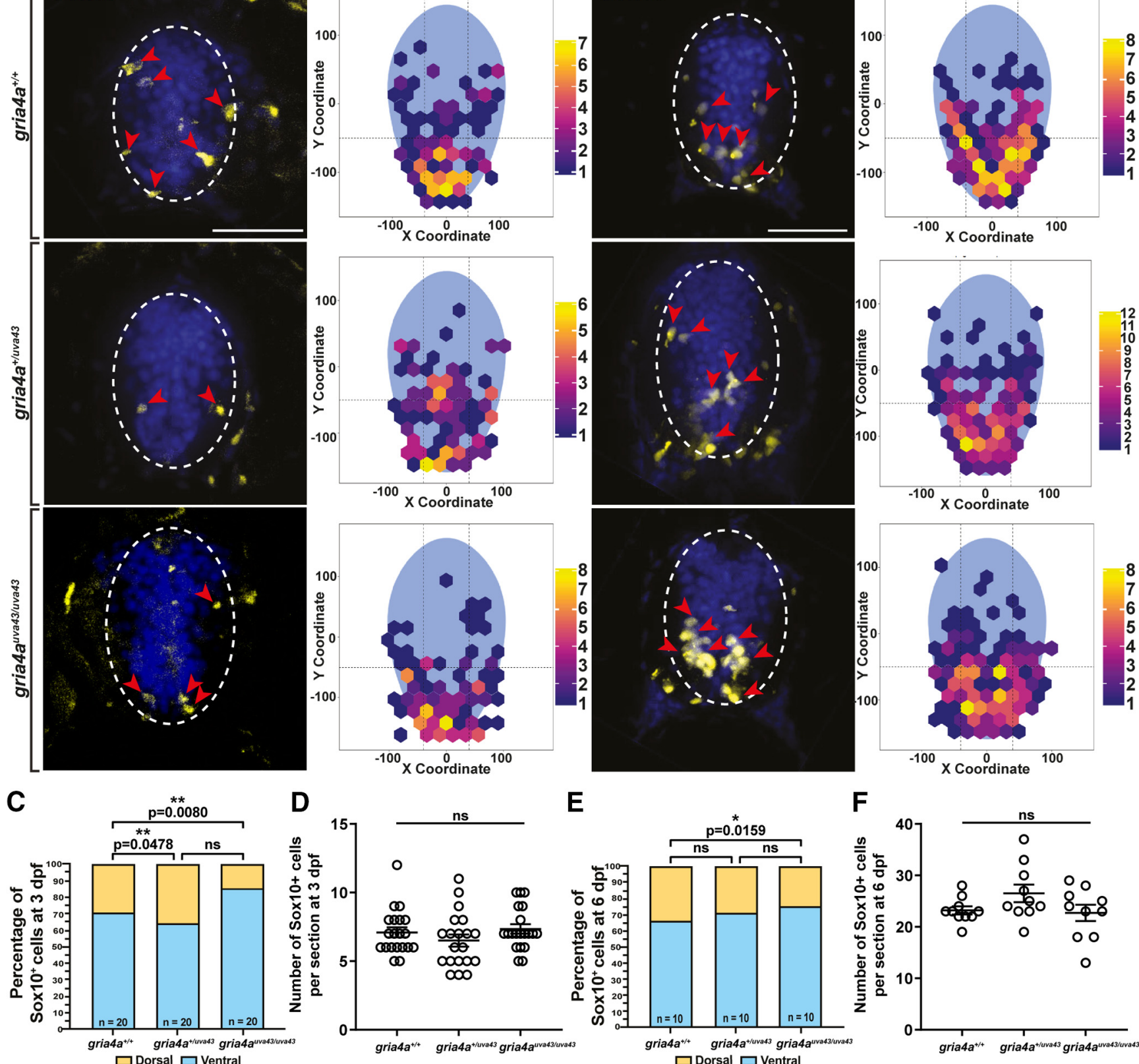

Figure 5. gria4a mutants have altered OLC distribution, but not total number. $A, B$, Heatmaps and representative images of Sox 10 antibody labeling and DAPI staining in 3- and 6-dpf spinal cord sections from gria4a ${ }^{+/+}(3 \mathrm{dpf} n=20,6 \mathrm{dpf} n=10)$, gria4a ${ }^{+/ \text {wva43 }}$ ( $\left.3 \mathrm{dpf} n=21,6 \mathrm{dpf} n=10\right)$, and gria4a $a^{\text {uva43/uva43 }}(3 \mathrm{dpf} n=20,6 \mathrm{dpf} n=10)$ larvae. Red arrowheads denote Sox $10^{+}$OLCs. Dashed circle delineates the edge of the spinal cord. In accompanying panels for each genotype and age, we plot the data as a heatmap, with warmer colors representing higher numbers of Sox $10^{+}$cells. Blue ovals represent an average of the size of all transverse spinal cord sections analyzed, and each hexagon represents a Sox $10^{+}$cell counted. Caution was taken to only count cells within the spinal cord boundary for each section, and points on the heatmap that fall outside of the average blue oval spinal cord were in fact, located in the spinal cord in the

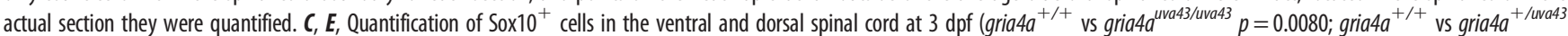
$p=0.0478$ ) and $6 \mathrm{dpf}\left(\right.$ gria $4 a^{+/+}$vs gria4ava43/uva43 $\left.p=0.0159\right)$. D, F, Quantification of the number of Sox $10^{+}$cells per section in each genotype at 3 and $6 \mathrm{dpf}$. ns $=$ no significant differences between groups. Scale bars: $50 \mu \mathrm{m}$.

into gria4a $a^{\text {uva } 43 / u v a 43} ; \operatorname{Tg}(m b p: e g f p-C A A X)$ host embryos at the same stage and used in vivo, time-lapse imaging to analyze the migration dynamics of transplanted OLCs between 56 and 72 hpf. As a control, we also transplanted cells from dextraninjected gria4a $a^{\text {uva } 43 / u v a 43} ; \operatorname{Tg}(m b p: e g f p-C A A X)$ embryos into gria $4 a^{\text {uva } 43 / \text { uva } 43} ; \operatorname{Tg}(m b p: e g f p-C A A X)$ host embryos to control for transplantation effects. At $56 \mathrm{hpf}, 66.7 \%$ of the transplanted

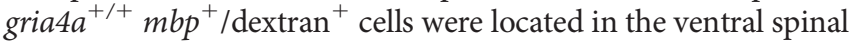
cord, and the remaining $33.3 \%$ were in the dorsal spinal cord, while all of the transplanted gria $4 a^{\text {uva } 43 / \text { uva } 43} \mathrm{mbp}^{+} /$dextran $^{+}$ cells were in the ventral spinal cord $(p=0.0191$; Fig. $6 F)$. At 72 hpf, $53.3 \%$ of the transplanted gria $4 a^{+/+} \mathrm{mbp}^{+} /$dextran $^{+}$cells were located in the ventral spinal cord, and the other $46.7 \%$ were located in the dorsal spinal cord, while $94.1 \%$ of the transplanted

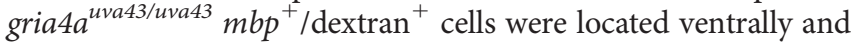
only $5.9 \%$ were located dorsally ( $p=0.0133$; Fig. $6 G$ ). The greater number of transplanted gria $4 a^{+/+}$OLCs in the dorsal spinal cord compared with the transplanted gria $4 a^{\text {uva } 33 / u v a 43}$ OLCs is 

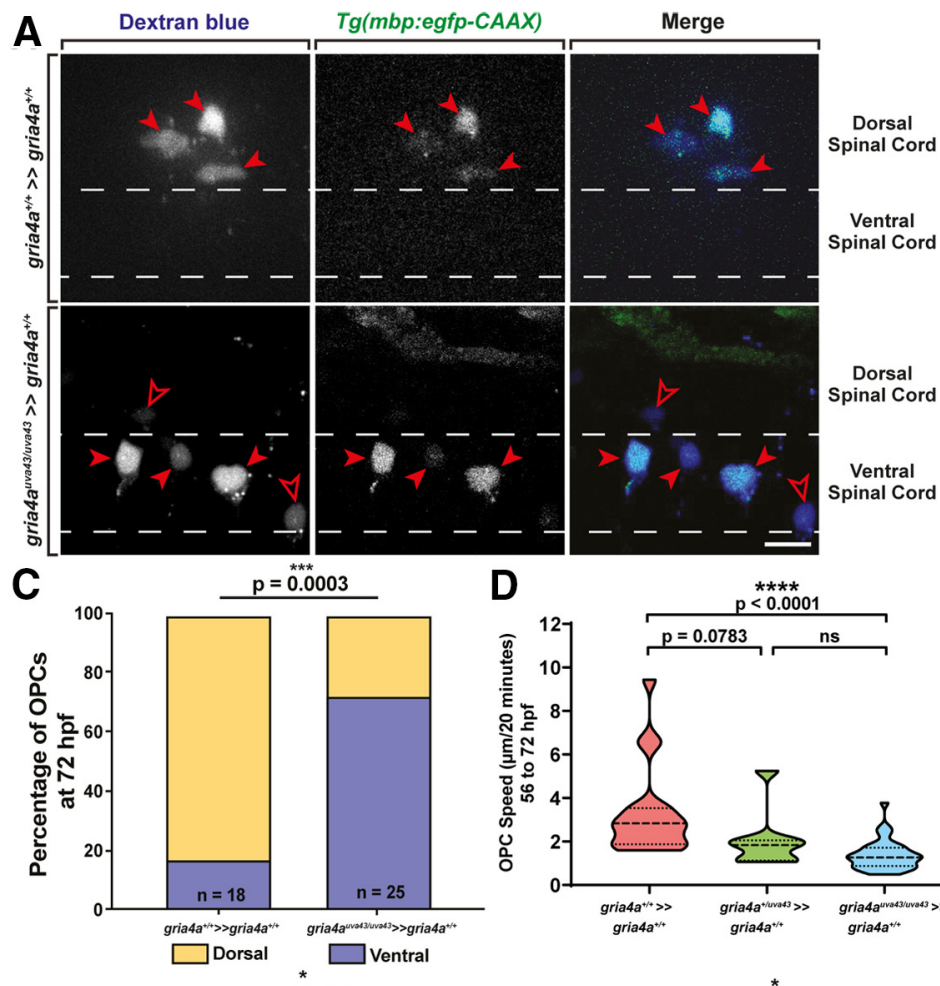

B
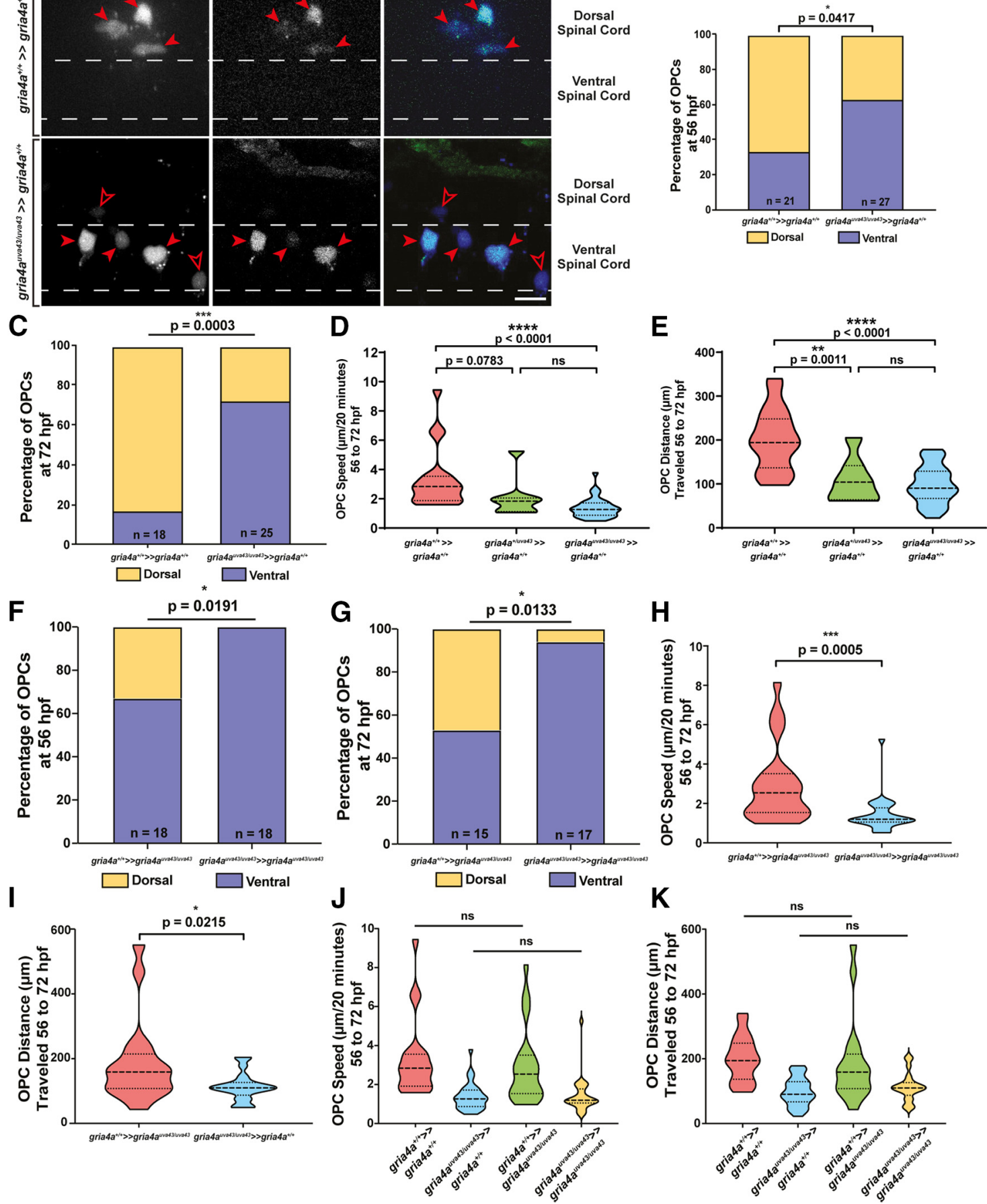

Figure 6. Mutation of gria4a alters OLC migration in a cell-autonomous manner. $A$, Genetic mosaic embryos with $\mathrm{Tg}$ (mbp:egfp-CAAX);gria4a ${ }^{+/+}$and gria4ava43/uva43 cells transplanted into

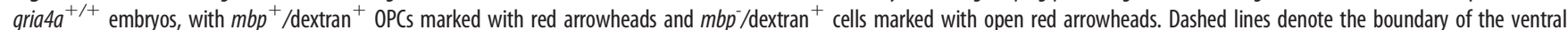
spinal cord. B, C, Quantification of the number of transplanted OLCs in the dorsal versus ventral spinal cord for gria4a ${ }^{+/+}$( $n=21$ at $56 \mathrm{hpf}$ and 18 at $72 \mathrm{hpf}$ ) and gria4ava43/uva43 ( $n=27$ at

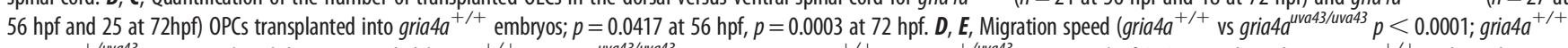

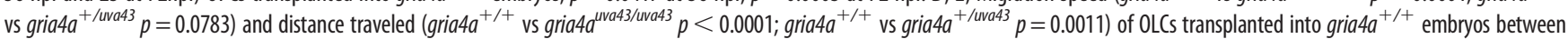

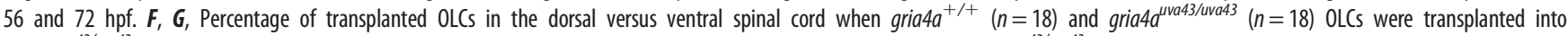

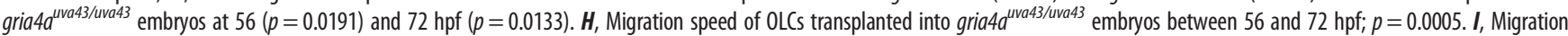
distance of $0 \mathrm{LCS}$ transplanted into gria4a $a^{\text {uva } 43 / \text { uva } 43}$ embryos between 56 and $72 \mathrm{hpf} ; p=0.0215$. J, Comparison of migration speed of gria4a $a^{+/+}$and gria $4 a^{\text {uva } 43 / \text { /va } 43}$ 0LCs transplanted into gria4a $^{+/+}$and gria4ava43/uva43 embryos between 56 and $72 \mathrm{hpf} ; p=0.4745$ for gria4a $a^{+/+}$into gria4a $a^{\text {uva } 43 / \text { /va } 43}$ versus gria4a $a^{+/+}$into gria4a $a^{+/+}, p=0.7859$ for gria4ava43/uva43 into

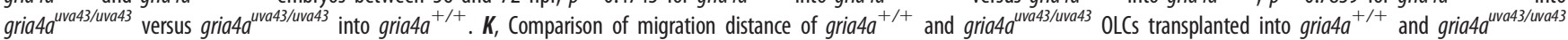

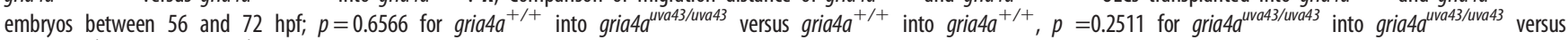

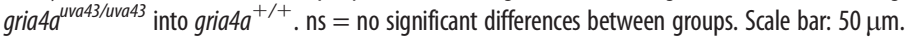


A

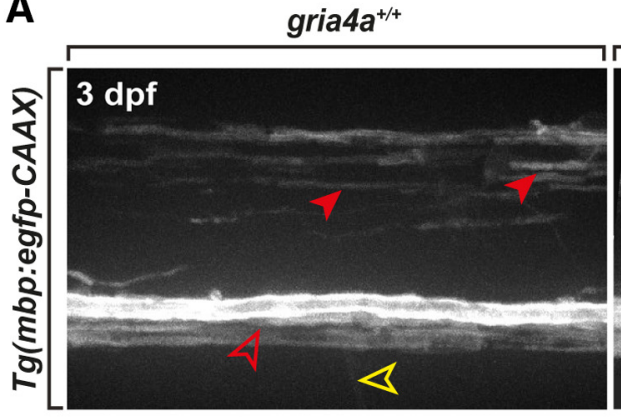

B
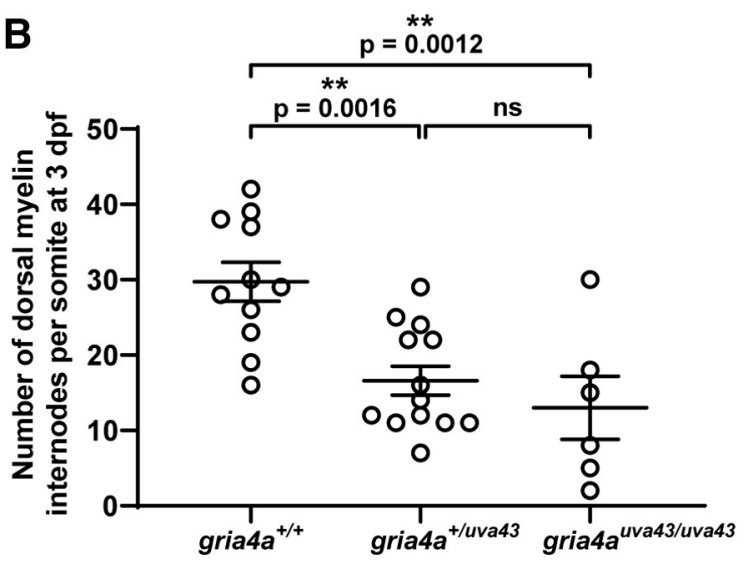

D

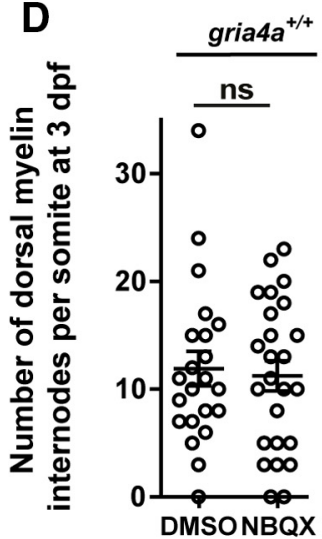

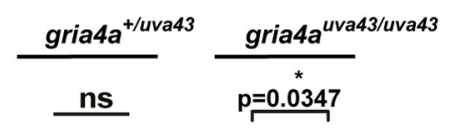
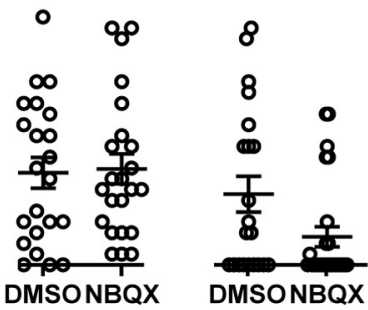

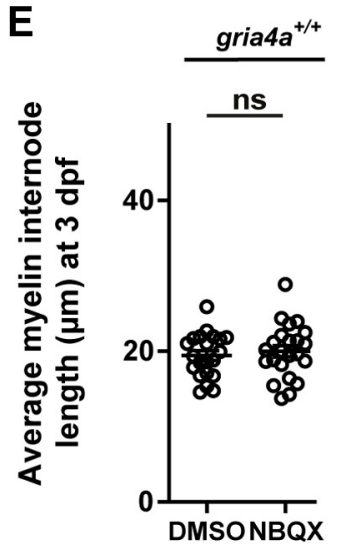

gria4a $a^{+/ u v a 43}$

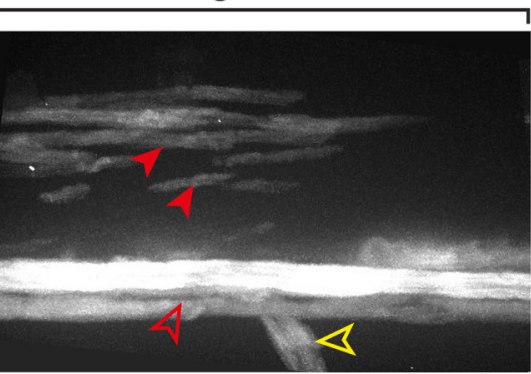

C

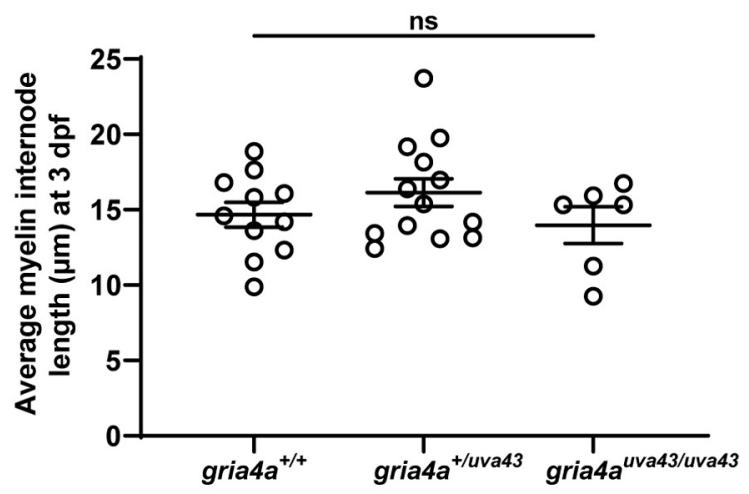

E

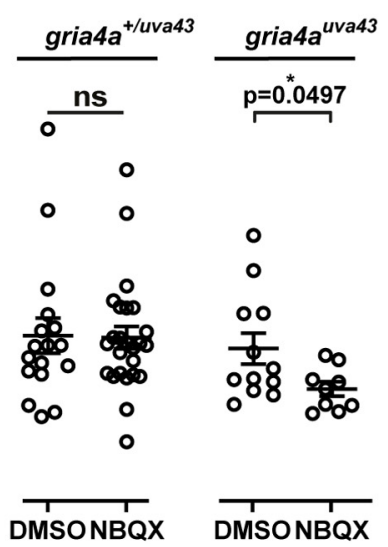

Figure 7. Dorsal spinal cord myelination is reduced in gria4a $a^{\text {uva43/uva43 }}$ larvae at $3 \mathrm{dpf}$. $A$, Myelin expression marked by $\operatorname{Tg}\left(\right.$ mbp:egfp-CAAX) in gria4a $a^{+/+}(n=11)$, gria4a $a^{+/ \text {uva43 }}(n=12)$, and gria4a $a^{\text {uva } 43 / \text { uva43 }}(n=6)$ larvae at $3 \mathrm{dpf}$, with myelin internodes labeled by red arrowheads. Yellow arrowheads denote normal peripheral myelin along spinal motor nerve roots. $\boldsymbol{B}$, Mean \pm SEM of the number of dorsal myelin internodes per somite at $3 \mathrm{dpf}$ in each genotype, measured in z-stack; $p=0.0003$ across all groups (gria4a $a^{+/+}$vs gria4a $a^{\text {uva } 43 / \text { wva43 }} p=0.0012$; gria $4 a^{+/+}$vs gria $\left.4 a^{+/ \text {/va } 43} p=0.0016\right)$. C, Quantification of the average myelin internode length for each genotype at $3 \mathrm{dpf}$. $\boldsymbol{D}$, $\boldsymbol{E}$, Mean \pm SEM of the number $(\boldsymbol{D})$ and average length $(\boldsymbol{E})$ of dorsal myelin internodes per somite at $3 \mathrm{dpf}$ in Tg(mbp:egfp-CAAX) larvae treated with $40 \mu \mathrm{m} \mathrm{NBQX}$ in 1\% DMSO or 1\% DMSO alone from 55 to $80 \mathrm{hpf}$. gria4a ${ }^{+/+} n=22$ and 25 ,

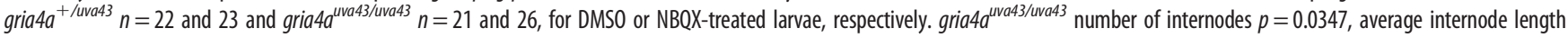
$p=0.0497$. ns $=$ no significant differences between groups. Scale bar: $25 \mu \mathrm{m}$.

consistent with the migration effects we observed both with gria $4 a^{\text {uva } 43 / \text { uva } 43}$ OLCs transplanted into gria $4 a^{+/+}$embryos and global gria $4 a^{u v a 43 / u v a 43}$ embryos, which provides evidence for this altered distribution pattern as a cell-autonomous phenomenon. However, we did observe a greater proportion of dorsal OLCs when cells were transplanted into gria $4 a^{+/+}$embryos compared with cells transplanted into gria $4 a^{\text {uva } 43 / u v a 43}$ embryos, which indicates that there may be a minor non-cell-autonomous effect also influencing the distribution of OLCs in the developing spinal cord.

To further explore this non-cell-autonomous effect of loss of GluR4A on OLC migration, we analyzed the migration dynamics of OLCs transplanted into gria $4 a^{\text {uva } 43 / \text { uva } 43}$ mutant host embryos from 56 to $72 \mathrm{hpf}$ using the Manual Tracking plugin in ImageJ. Wild-type OLCs transplanted from gria $4 a^{+/+}$embryos into mutant hosts migrated faster $(p=0.0005$; Fig. $6 H)$ and for a greater distance $(p=0.0215$; Fig. $6 I)$ than mutant OLCs transplanted from gria $4 a^{\text {uva } 43 / \text { uva } 43}$ embryos into mutant host embryos between 56 and $72 \mathrm{hpf}$ (Fig. 6H,I). These differences in migration dynamics were similar to those observed in both the global gria $4 a^{\text {uva } 43 / \text { uva } 43}$ migration analysis and the transplant into wildtype hosts. To determine the contributions of cell-autonomous versus non-cell-autonomous effects on these migration dynamics, we compared the results from OLCs transplanted into either 
wild-type or mutant host embryos. The migration speed of gria4a $a^{\text {uva } 43 / \text { uva } 43}$ OLCs transplanted into gria $4 a^{\text {uva } 43 / \text { uva } 43}$ embryos was similar to that of gria $4 a^{\text {uva43/uva43 }}$ OLCs transplanted into gria $4 a^{+/+}$embryos $(p=0.7859$; Fig. $6 J)$, and the speed of ${\text { gria } 4 a^{+/+}}_{\text {OLCs transplanted into gria } 4 a^{\text {uva } 43 / \text { uva } 43} \text { embryos }}$ was similar to that of $g r i a 4 a^{+/+}$OLCs transplanted into gria $4 a^{+/+}$embryos $(p=0.4745$; Fig. $6 J)$. Furthermore, the distance traveled by gria $4 a^{\text {uva43/uva43 }}$ OLCs transplanted into gria $4 a^{\text {uva } 43 / \text { uva } 43}$ embryos was similar to that of gria $4 a^{\text {uva } 43 / u v a 43}$ OLCs transplanted into gria $4 a^{+/+}$embryos $(p=0.2511$; Fig. $6 K)$, and the distance migrated by gria $4 a^{+/+}$OLCs transplanted into gria4a $a^{\text {uva } 43 / \text { uva } 3}$ embryos was similar to that of ${\text { gria } 4 a^{+/+}}$OLCs transplanted into gria $4 a^{+/+}$embryos $(p=0.6566$; Fig. $6 K)$. Together, these results show that gria4a cell-autonomously drives OLC migration speed and distance, with some possible minor contributions of non-cell-autonomous effects to the dorsoventral distribution of OLCs.

\section{Mutation of gria4a reduces myelination in the dorsal spinal cord}

Several recent studies demonstrate that oligodendrocyte myelination of CNS axons can be activity dependent (Hines et al., 2015; Koudelka et al., 2016; de Faria et al., 2019; Noori et al., 2020). Therefore, we hypothesized that the combination of reduced dorsal OPC migration and reduced sensitivity of OLCs to neuronal activity because of mutation of gria $4 a$ would lead to a reduction in dorsal spinal cord myelination in mutant larvae. To investigate this hypothesis, we used the $T g(m b p: e g f-C A A X)$ transgenic line to perform in vivo imaging at $3 \mathrm{dpf}$ when myelination is occurring in the developing spinal cord. From the z-stacks of these images, we quantified the number of myelin internodes in the dorsal spinal cord and measured the internode length in both mutant and wild-type larvae (Fig. $7 A-C$ ). From these studies, we observed fewer myelin internodes in both gria $4 a^{+/ \text {uva } 43}$ and

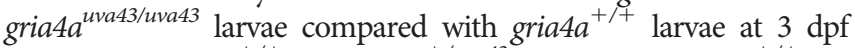

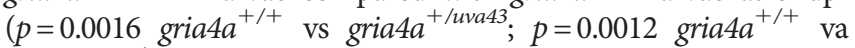
gria4a ${ }^{\text {uva } 43 / \text { uva } 43}$; Fig. $\left.7 A, B\right)$, although the average internode length was similar across genotypes $(p=0.3008$; Fig. $7 A, C)$. Notably, the slightly larger number of dorsal OPCs in heterozygous larvae at 3 dpf (Fig. 5A,C) may indicate some compensation in the heterozygous larvae for the decrease in myelination we observed.

Because we observed an interesting migration result when treating gria4a mutant larvae with NBQX, we hypothesized that it may also affect myelination in mutant larvae. To investigate this, we treated $\operatorname{Tg}($ olig2:dsred $) ; \operatorname{Tg}(n k x 2.2 a$ :megfp) embryos from 55 to $80 \mathrm{hpf}$, which is after most OLCs have migrated out of the ventral spinal cord, with either $40 \mu \mathrm{M}$ NBQX dissolved in $1 \%$ DMSO or with $1 \%$ DMSO as a control. We then performed in vivo imaging and compared dorsal myelination between the different genotypes. Blocking AMPAR signaling with NBQX from 55 to $80 \mathrm{hpf}$ did not significantly change the number of myelin internodes per somite, nor affected the average length per internode, in wild-type and heterozygous larvae (Fig. $7 D, E$ ). However, treatment with NBQX decreased the internode length as well as number of dorsal myelin internodes in gria $4 a$ mutant larvae (number of internodes $p=0.0347$, internode length $p=0.0497$; Fig. $7 D, E)$. Therefore, while NBQX blocks OPC migration in wild-type larvae (Fig. 3), it did not significantly alter myelination. On the other hand, NBQX increased OPC migration in gria4a mutant larvae (Fig. 3), while it significantly decreased myelination.

We also imaged myelin at $5 \mathrm{dpf}$ in the $T g(m b p: e g f p-C A A X)$ transgenic line, to assess whether the decreased myelination was caused by a delay in development. Because of increased myelination at this stage, our measurements of myelin internode number and length were conducted in a single z-plane for all groups. At 5 $\mathrm{dpf}$, the number of myelin internodes remained significantly reduced in gria $4 a^{\text {uva } 3 / \text { uva } 43}$ larvae compared with gria $4 a^{+/+}$larvae $\left(p<0.0001\right.$ gria $4 a^{\text {uva } 43 / \text { uva } 43}$; Fig. $\left.8 A, B\right)$, while the number of internodes in gria $4 a^{+/ u v a 43}$ was now significantly larger than in gria4a $a^{\text {uva43/uva43 }}$ larvae ( $p=0.0027$; Fig. $\left.8 A, B\right)$, although still not completely at the same level as observed in gria $4 a^{+/+}$larvae. The average internode length was not significantly different between the groups $(p=0.8968$; Fig. $8 C)$. Therefore, we conclude that both the decrease in dorsal OPC migration and myelination in gria $4 a^{\text {uva43/uva43 }}$ larvae were not caused by a developmental delay. This is in line with our observation that mutant larvae appear healthy and indistinguishable from wild-type and heterozygous larvae with regard to body length and eye size both at 2 and $5 \mathrm{dpf}$ (Fig. 1F). Interestingly, at both 3 and $5 \mathrm{dpf}$, the gria $4 a^{+/ \text {uva } 43}$ myelin phenotypes were either intermediate between the gria $4 a^{+/+}$and gria $4 a^{\text {uva } 43 / \text { uva } 43}$ phenotypes, or comparable to the gria $4 a^{\text {uva } 43 / \text { uva } 43}$ phenotype. However, the intermediate myelin phenotype in gria $4 a^{+/ u v a 43}$ larvae was independent of a significant change in dorsal OPC distribution compared with gria $4 a^{+/+}$larvae. This leads us to hypothesize that GluR4A may affect migration and myelination via distinct mechanisms, which we will address in more detail in the discussion.

In the previous set of studies, we used the stable $\mathrm{Tg}(\mathrm{mbp}$ :egfp$C A A X)$ line, which makes it is difficult to distinguish internodes from individual oligodendrocytes. Therefore, to assess myelin on an individual cell basis, we injected the mbp:egfp-CAAX construct into gria $4 a^{+/+}$, gria $4 a^{+/ u v a 43}$, and gria $4 a^{\text {uva } 43 / \text { uva } 43}$ larvae and imaged individual oligodendrocytes at $5 \mathrm{dpf}$. We observed that although the average length per internode of dorsal oligodendrocytes was comparable between the different genotypes (Fig. 8D,E), gria $4 a^{\text {uva } 43 / u v a 43}$ oligodendrocytes made more internodes per cell compared with gria $4 a^{+/+}$oligodendrocytes $(p=0.0188$; Fig. $8 D, F)$. Interestingly, there was no difference in the number of internodes or average internode length per cell in ventral oligodendrocytes across the different genotypes (Fig. $8 D, G, H$ ).

\section{Glutamate signaling via AMPARs regulates OPC migration and myelination by modulating voltage-gated calcium channels}

Several proposed mechanisms for AMPAR-mediated glutamate signaling as a regulator of OPC development involve downstream $\mathrm{Ca}^{2+}$ influx via voltage-gated calcium channels ( $\mathrm{Gudz}$ et al., 2006; Paez et al., 2010; Harlow et al., 2015). To determine whether voltage-gated calcium channel activation plays a role in directing OPC migration, we treated gria4a mutant, heterozygous, and wild-type $\mathrm{Tg}$ (olig2:dsred); $\mathrm{Tg}(\mathrm{mbp}$ :egfp-CAAX) embryos with either $5 \mu \mathrm{M}$ the L-type voltage-gated calcium channel agonist ( \pm )-Bay K 8644 in 1\% DMSO or 1\% DMSO alone from 55 to $72 \mathrm{hpf}$ and performed in vivo, time-lapse imaging. Both wild-type and mutant larvae treated with $( \pm$ )-Bay K 8644 looked healthy and indistinguishable from DMSO-treated larvae (Fig. 9A). At 56 hpf, we observed a similar number of dorsal OPCs across all genotypes and conditions $(p=0.4721$; Fig. 9B). In contrast, at $72 \mathrm{hpf}$, we observed fewer dorsal OPCs in gria $4 a^{u v a 43 / u v a 43}$ embryos treated with DMSO compared with $g r i a 4 a^{+/+}$embryos treated with DMSO $(p=0.0008$; Fig. $9 C)$. However, in gria $4 a^{u v a 43 / u v a 43}$ embryos treated with ( \pm )-Bay K 8644, there were more dorsal OPCs at $72 \mathrm{hpf}$ compared with the gria $4 a^{u v a 43 / u v a 43}$ DMSO control 
A
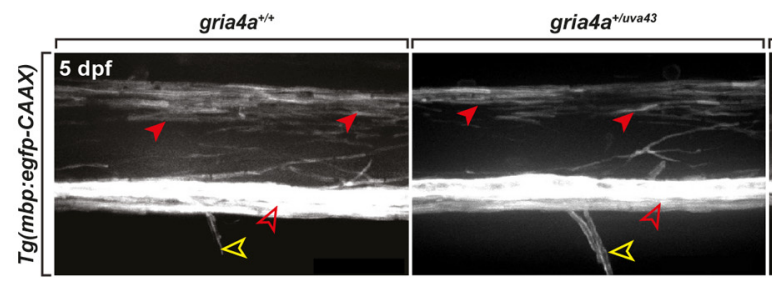

C

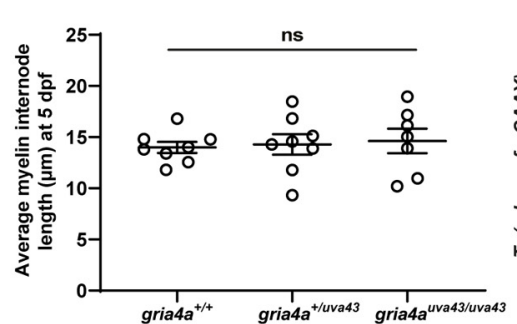

F

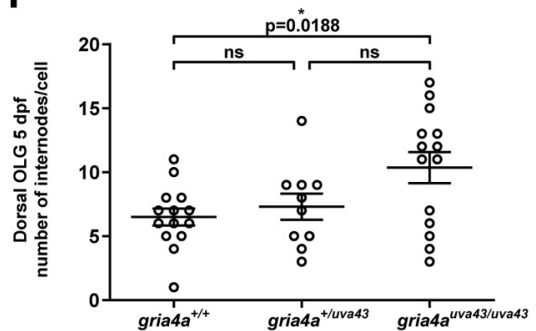

D

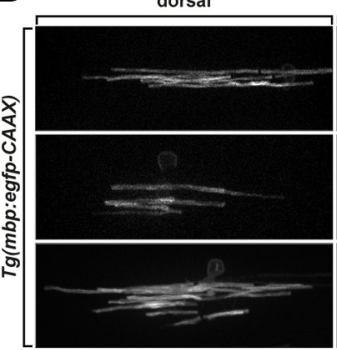

G

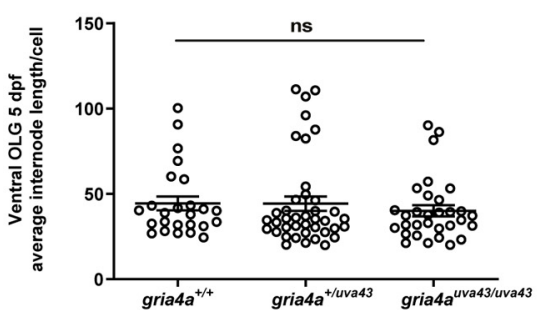

ventral
B
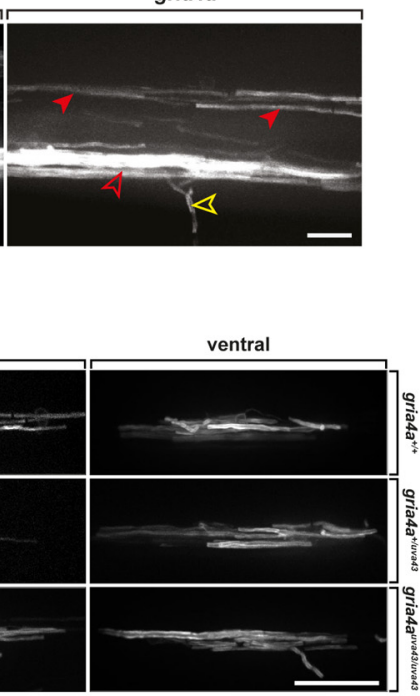

E

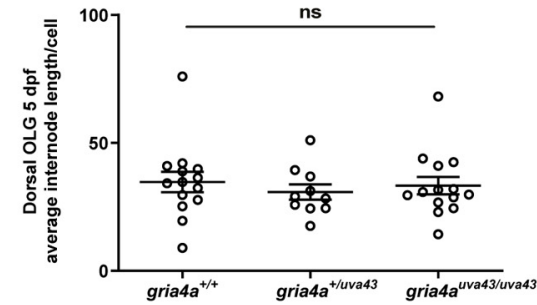

H

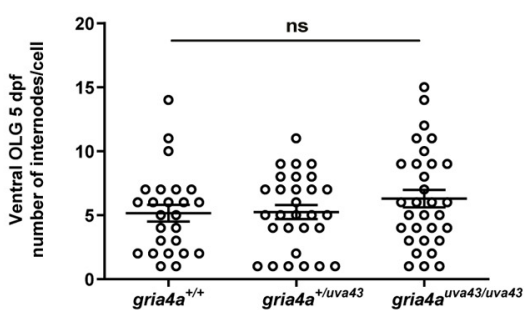

Figure 8. gria4a $a^{u v a 43 / u v a 43}$ larvae have less dorsal myelin at $5 \mathrm{dpf}$, but more internodes per cell. $A$, Myelin expression marked by $T g(m b p: e g f p-C A A X)$ in gria4a $a^{+/+}(n=8), g r i a 4 a^{+/ u v a 43}$ $(n=8)$, and gria $4 a^{u v a 43 / u v a 43}(n=7)$ larvae at $5 \mathrm{dpf}$, with myelin internodes labeled by red arrowheads. Yellow arrowheads denote normal peripheral myelin along spinal motor nerve roots. $\boldsymbol{B}$, Mean \pm SEM of the number of myelin internodes per somite at $5 \mathrm{dpf}$ in each genotype in a single z-plane with the most myelin internodes visible (gria4a ${ }^{+/+}$vs gria4a $a^{\text {uva } 43 / \text { uva } 43}$ $p<0.0001 ;$ gria $4 a^{+/ u v a 43}$ vs gria $\left.4 a^{\text {uva } 43 / \text { uva } 43} p=0.0027\right)$. C, Mean \pm SEM of the average myelin internode length for each genotype at 5 dpf. $\boldsymbol{D}$, Representative images of myelin expression by individual oligodendrocytes marked by transient injections of the mbp:egfp-CAAX construct in gria $4 a^{+/+}$(ventral $n=25$ cells in $n=8$ larvae; dorsal $n=14$ cells in $n=6$ larvae),

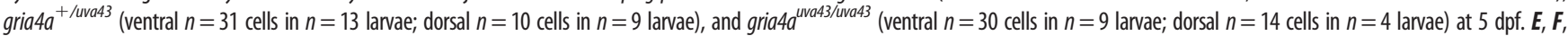
Mean \pm SEM of the number $(\boldsymbol{E})$ and average length $\left(\boldsymbol{F} ;{\text { gria } 4 a^{+/+}}\right.$vs gria4a $\left.a^{\text {uva43/uva43 }} p=0.0188\right)$ of ventral myelin internodes per somite at 5 dpf in each genotype. $\boldsymbol{G}, \boldsymbol{H}$, Mean \pm SEM of the number $(\boldsymbol{G})$ and average length $(\boldsymbol{H})$ of dorsal myelin internodes per somite at $5 \mathrm{dpf}$ in each genotype. ns $=$ no significant differences between groups. Scale bars: $25 \mu \mathrm{m}(\boldsymbol{A})$ and $50 \mu \mathrm{m}(\boldsymbol{D})$.

group $(p<0.0001$; Fig. 9C), and there was a similar number of dorsal OPCs in both the gria $4 a^{+/+}( \pm)$-Bay K 8644-treated group $(p=0.5983)$ and the gria $4 a^{+/+}$DMSO control group $(p=0.9578)$. These results indicate $\mathrm{Ca}^{2+}$ signaling induces OPC migration downstream of AMPAR activation (Fig. 9D), and that stimulation of voltage-gated calcium channels with (士)-Bay K 8644 effectively rescues the OPC migration phenotype in gria $4 a^{u v a 43 / u v a 43}$ embryos. Notably, OPC migration in wild-type larvae did not increase with ( \pm )-Bay K 8644 treatment, whereas migration of OPCs in gria $4 a^{u v a 43 / u v a 43}$ larvae did. We hypothesize this is because wild-type OPCs have already reached a maximum threshold of $\mathrm{Ca}^{+}$influx that drives OPC migration under physiological conditions.

Similar to its effect on OPC migration, we hypothesized that voltage-gated calcium channel activation with ( \pm )-Bay K 8644 would rescue the myelin phenotype in gria $4 a$ mutant larvae. To assess this, we treated gria4a wild-type, heterozygous, and mutant $\mathrm{Tg}(\mathrm{mbp}$ :egfp-CAAX) embryos with either $5 \mu \mathrm{M}( \pm)$-Bay $\mathrm{K}$ 8644 in $1 \%$ DMSO or $1 \%$ DMSO alone from 55 to $72 \mathrm{hpf}$ or from 55 to $80 \mathrm{hpf}$, and performed in vivo imaging of myelin at 80 hpf. In gria $4 a^{+/+}$larvae, treatment with ( \pm )-Bay K 8644 did not alter the number or the length of myelin internodes (Fig. $10 A-C)$. In gria4a $a^{+/ u v a 43}$ larvae, ( \pm )-Bay K 8644 also did not significantly change the number of myelin internodes (Fig. 10A, $D)$. However, the average internode length was slightly decreased on a 24 -h treatment $(p=0.0371$; Fig. $10 A, E)$. Interestingly, in gria $4 a^{\text {uva } 3 / \text { uva } 43}$ larvae treated for $24 \mathrm{~h}$, but not $16 \mathrm{~h}$, with $( \pm)$-Bay K 8644, we observed a significant increase in the number of dorsal myelin internodes $(p=0.0192$; Fig. $10 A, F)$, while the internode length was not altered (Fig. 10A,G). Therefore, while OPC migration is restored after $16 \mathrm{~h}$ of voltage-gated calcium channel activation with ( \pm )-Bay K 8644 (Fig. 9B), voltagegated calcium channel activation is also required for efficient myelination, as only a 24-h treatment with ( \pm )-Bay K 8644 rescued the myelin phenotype in gria $4 a$ mutant larvae.

\section{Discussion}

During development, spinal cord OPCs must migrate extensively from their origin in the ventral spinal cord to distribute evenly throughout the CNS and myelinate axons. Several studies have examined how OPC AMPAR activation may stimulate this migration (Gudz et al., 2006; Paez et al., 2010), but in vivo experiments have yielded conflicting results (Harlow et al., 2015; Kougioumtzidou et al., 2017). One study demonstrated in vitro that in wild-type mouse 
A
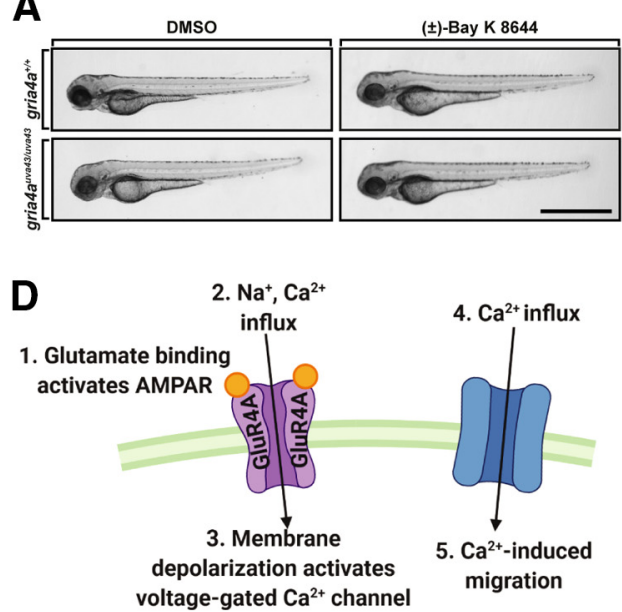

B

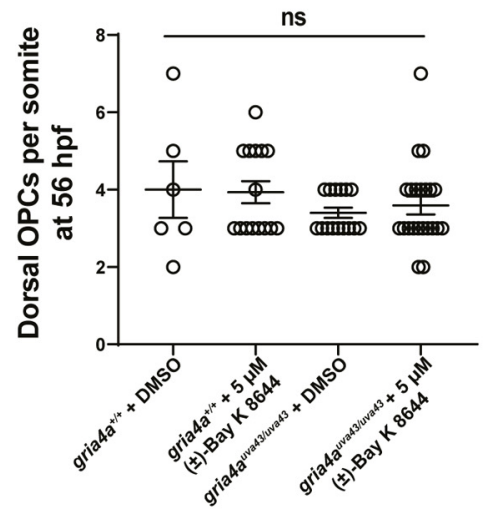

C

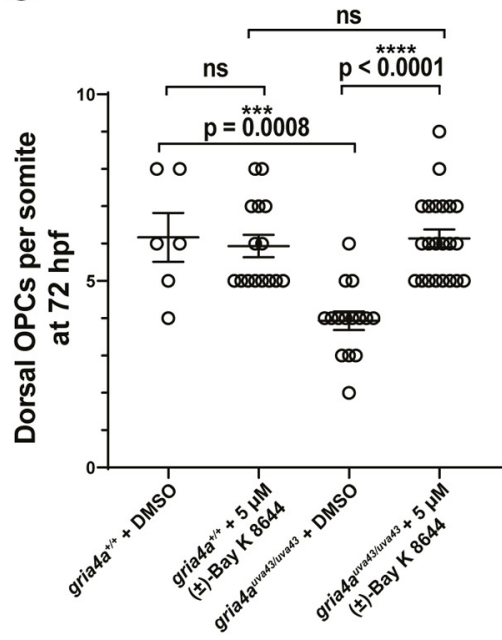

Figure 9. Activation of voltage-gated $\mathrm{Ca}^{2+}$ channels restore normal OPC migration in gria4a ${ }^{\text {uva43/uva43 }}$ larvae. $\boldsymbol{A}$, Brightfield images of 80 -hpf gria4a wild-type and mutant larvae after treatment with $5 \mu \mathrm{m}$ voltage-gated calcium channel agonist ( \pm )-Bay K 8644. B, Mean \pm SEM of the number of dorsal OPCs per somite in gria4a $a^{+/+}$and gria4ava43/uva43 embry0s at 56 hpf following treatment with either $5 \mu \mathrm{M}( \pm)$-Bay K 8644 (gria4a $a^{+/+} n=15$, gria $\left.4 a^{\text {uva } 43 / \text { uva } 43} n=22\right)$ in $1 \%$ DMSO or $1 \%$ DMSO control (gria $4 a^{+/+} n=6$, gria $\left.4 a^{\text {uva } 43 / u v a 43} n=15\right) ; p=0.4721 . C_{\text {, }}$ Mean \pm SEM of the number of dorsal OPCs per somite in gria $4 a^{+/+}$and gria4a ${ }^{\text {uva } 43 / \text { uva } 43}$ larvae at 72 hpf following treatment with either $5 \mu \mathrm{m}( \pm)$-Bay $\mathrm{K} 8644$ in $1 \%$ DMSO or $1 \%$ DMSO control. $p<0.0001$ across all groups, $p=0.5983$ for gria4a $a^{\text {uva } 3 / \text { uva } 43}+( \pm)$-Bay $\mathrm{K} 8644$ versus gria4a $a^{+/+}+( \pm)$-Bay $\mathrm{K} 8644, p=0.0008$ for gria4a $a^{\text {uva } 43 / \text { uva } 43}+$ DMSO versus gria $4 a^{+/+}+$DMSO, and $p<0.0001$ for gria4a $a^{\text {uva43/uva43 }}+( \pm)$-Bay K 8644 versus gria4a $a^{\text {uva43/uva43 }}+$ DMSO. D, Model of mechanism through which GluR4A works with voltage-gated calcium channels to regulate $\mathrm{OPC}$ migration. $\mathrm{ns}=$ no significant differences between groups. Scale bar: $1 \mathrm{~mm}$.

OPCs, AMPA accelerates OPC migration. Notably, AMPA treatment resulted in internalization of the calcium-impermeable GluR2 subunit and increased $\mathrm{Ca}^{2+}$ influx. However, in ex vivo cerebellar slices from GluR2-null mouse, OPCs did not accelerate in response to AMPAR stimulation and resulted in reduced baseline $\mathrm{Ca}^{2+}$ influx in OPCs, possibly because of reduced neuronal activity (Harlow et al., 2015). The authors describe this effect on migration as the result of complex formation between PLP, $\alpha \mathrm{v}$ integrin, and the GluR2 subunit, which does not occur in the absence of GluR2 (Harlow et al., 2015). Thus, GluR2 was required for complex formation and initiation of migration followed by internalization of GluR2 and an increase in $\mathrm{Ca}^{2+}$ influx. Although not discussed in detail by the authors, GluR4 was also bound by to PLP complex. Our results reveal similar effects on OPC migration following GluR4A disruption, suggesting that these effects are not specific to GluR2 and that they may both act via AMPAR-mediated $\mathrm{Ca}^{2+}$ influx, not solely through PLPintegrin complex formation. We conclude from our studies that GluR4A is an important AMPAR subunit for OPC migration and provide evidence for neuron-OPC AMPAR signaling that directly affects OPC migration before myelination in vivo.

\section{AMPAR subunit composition modulates calcium signaling in OPCs}

Several studies have examined the activation of voltage-gated calcium channels as downstream mediators of AMPAR-mediated membrane depolarization in OPCs as a possible driver of developmental processes including membrane extension and myelination (Yuan et al., 1998; Butt, 2006; Gallo and Armstrong, 2008; Giesen et al., 2020). Following voltage-gated calcium channelmediated $\mathrm{Ca}^{2+}$ influx into OPCs, numerous pathways involved in migration are activated, including $\mathrm{PKC}$-dependent process extension, TrK activation, and PKA activation (Paez et al., 2010). In mice, the expression of voltage-gated calcium channels in OLCs varies with developmental stage; OPCs express these channels at a higher level, and have correspondingly higher $\mathrm{Ca}^{2+}$ currents than later stages of OLCs (Blankenfeld Gv et al., 1992).
This developmental variation suggests roles for voltage-gated calcium channel-mediated $\mathrm{Ca}^{2+}$ influx at specific points in OPC development, in line with our findings that $\mathrm{Ca}^{2+}$ influx stimulates OPC migration.

The types of AMPAR subunits expressed in each cell affect its $\mathrm{Ca}^{2+}$ influx. At $72 \mathrm{hpf}$, OLCs in the zebrafish spinal cord predominantly express two AMPAR subunits: gria $2 b$ and gria $4 a$ (Fig. 1A; Hoppmann et al., 2008). A posttranscriptional Q/R modification which makes mammalian GluR2 calcium impermeable is genetically encoded in the gria $2 b$ gene in zebrafish (Kung et al., 2001; Sobolevsky et al., 2003). Like other AMPAR subunits, gria $4 a$ is calcium-permeable, and the calcium permeability of each heterotetrameric AMPAR is dependent on its constituent subunits (Burnashev, 1998). In embryos harboring a mutation in gria4a, OPCs would likely have increased levels of AMPARs with a higher degree of calcium impermeability because of the relative increase in calcium-impermeable GluR2B subunits. In line with this, the loss of GluR4 in mature mouse oligodendrocytes leads to a decrease of $\mathrm{Ca}^{2+}$ influx (Evonuk et al., 2020). While in zebrafish some genetic compensation of other calcium-permeable subunits may occur, it is likely that gria4a ${ }^{\text {uva43/uva43 }}$ OPCs would have reduced $\mathrm{Ca}^{2+}$ influx following AMPAR stimulation compared with their wild-type counterparts. Notably, both in gria $4 a$ mutant and heterozygous larvae, we detected increased expression of the calcium impermeable gria $2 b$ subunit gene. Therefore, gria $4 a^{+/ u v a 43}$ OPCs would likely also have reduced $\mathrm{Ca}^{2+}$ influx following AMPAR stimulation compared with their wild-type counterparts, although more than in gria $4 a^{u v a 43 / \text { uva } 43}$ OPCs as they express one wild-type copy of gria $4 a$. This is in line with our observation that gria $4 a^{+/ u v a 43}$ OPCs exhibit an intermediate phenotype with regard to their migration speed and distance traveled.

\section{GluR4A cell autonomously drives OPC migration through glutamate sensing}

In our studies, transplanted gria $4 a^{\text {uva43/uva } 43}$ OPCs in wild-type larvae exhibited altered migration dynamics similar to those 
A
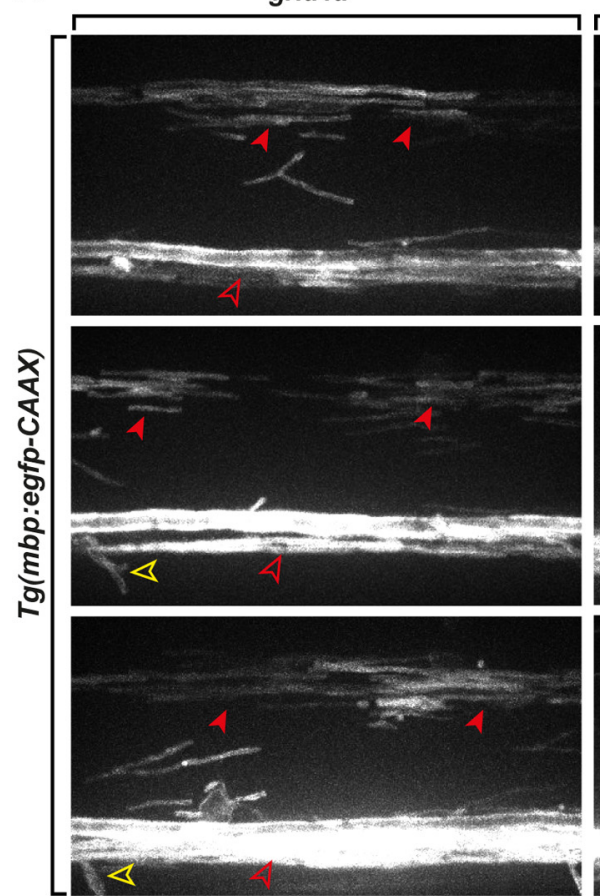

B

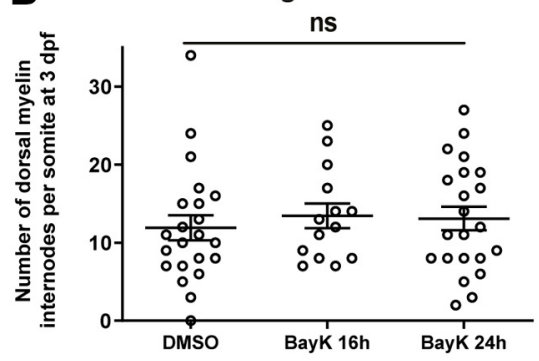

C

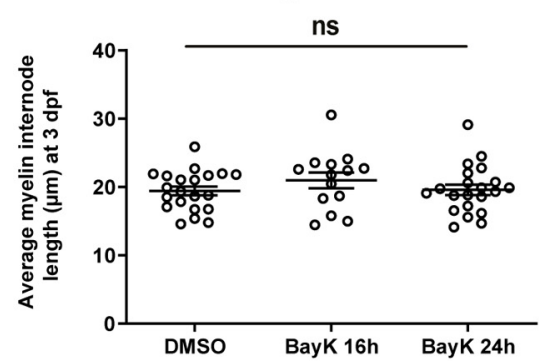

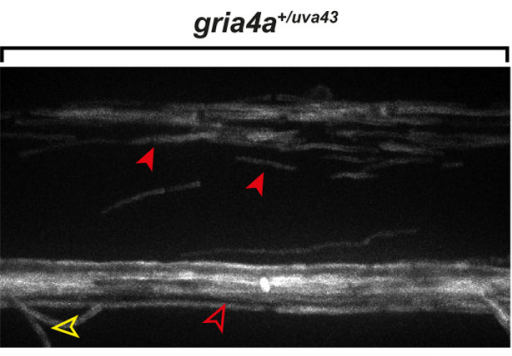
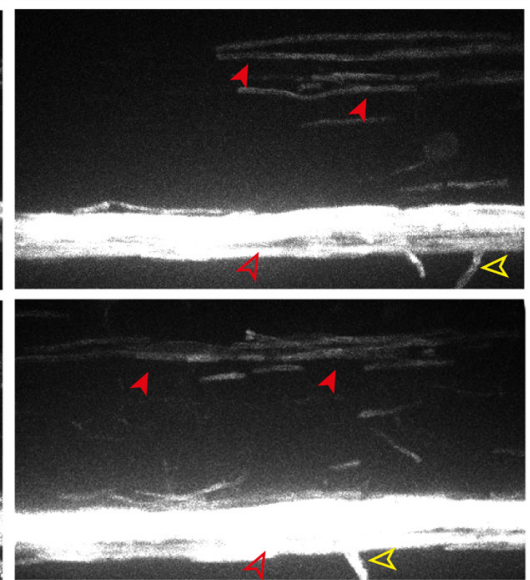

D

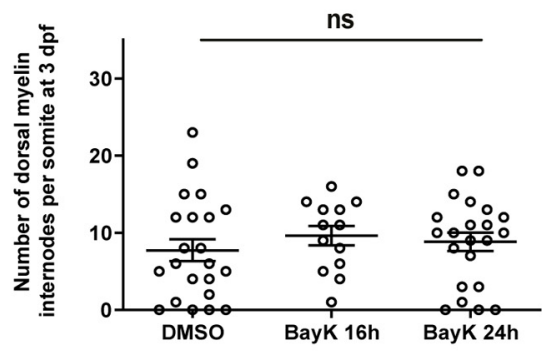

E

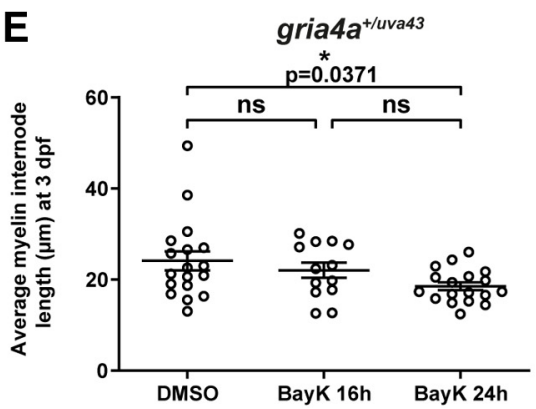

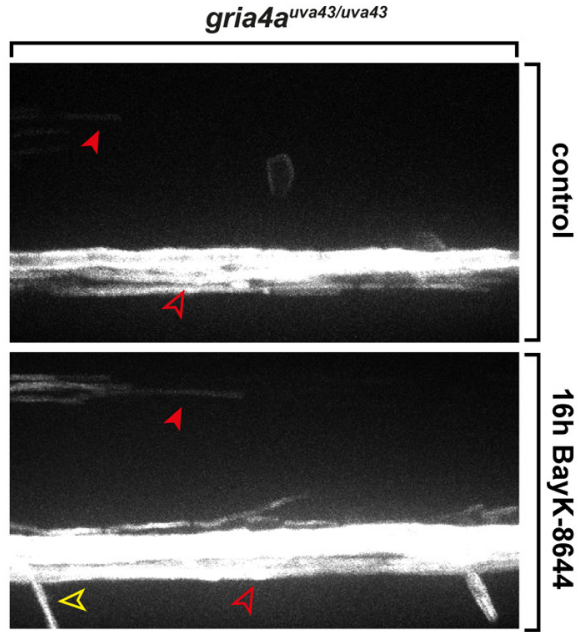

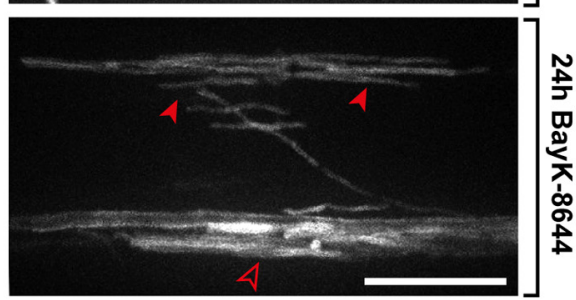

F

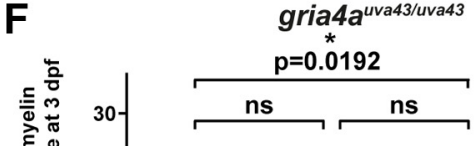

G

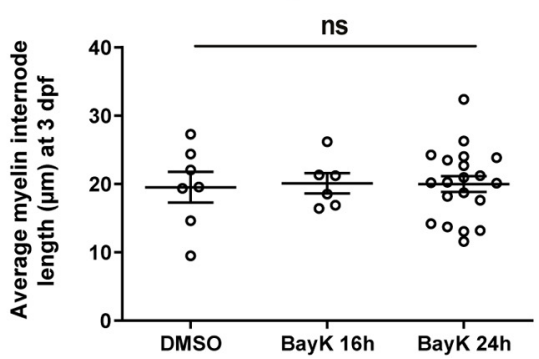

Figure 10. Activation of voltage-gated $\mathrm{Ca}^{2+}$ channels restore myelination after in gria4a ${ }^{\text {uva43/uva43 }}$ larvae. $A$, Representative images of myelin expression marked by $\mathrm{Tg}(\mathrm{mbp}$ :egfp-CAAX) in gria4a $^{+/+}(n=14-22)$, gria4a $^{+/ \text {/ua43 }}(n=13-22)$, and gria4a ${ }^{\text {uva43/uva43 }}(n=14-20)$ larvae at 3 dpf. Myelin internodes are labeled by red arrowheads. Yellow arrowheads denote normal peripheral myelin along spinal motor nerve roots. $\boldsymbol{B}, \boldsymbol{D}, \boldsymbol{F}$, Mean \pm SEM of the number of dorsal myelin internodes per somite at 3 dpf in gria4a ${ }^{+/+}(\boldsymbol{B})$, gria4a $a^{+/ \text {iva43 }}(\boldsymbol{D})$, and gria4ava43/uva43 $(\boldsymbol{F} ;$ DMSO vs BayK $24 \mathrm{~h} p=0.0192)$. C, $\boldsymbol{E}, \mathbf{G}$, Quantification of the average myelin internode length for at $3 \mathrm{dpf}$ in gria $4 a^{+/+}(\boldsymbol{C})$, gria4a ${ }^{+/ \text {wva43 }}(\boldsymbol{E}$; DMSO vs BayK $24 \mathrm{~h} p=0.0371)$, and gria4a $^{\text {uva43/uva43 }}(\boldsymbol{G}) . \mathrm{ns}=$ no significant differences between groups. Scale bar: $25 \mu \mathrm{m}$.

observed in global gria $4 a^{\text {uva } 43 / \text { uva } 43}$ larvae, indicating that OPC specific perturbation to GluR4A is sufficient to alter OPC migration in a cell-autonomous manner. Interestingly, gria $4 a^{+/ \text {uva } 3}$ OPCs that were transplanted into wild-type larvae also migrated slightly slower than when in global heterozygous larvae (Fig. 6). However, in gria $4 a^{+/ u v a 43}$ larvae, we observed a trend of reduced OPC migration speed, although it was not significant (Fig. 2). Therefore, it is not completely unexpected that we would see an intermediate phenotype when heterozygous OPCs were transplanted into wild-type hosts and were surrounded by a wild-type environment, which may cause the migration perturbations to heterozygous OPCs to become more obvious. Additionally, we found that wild-type OPCs transplanted into gria $4 a^{\text {uva } 3 / \text { uva } 43}$ embryos demonstrated the presence of mild, non-cell-autonomous effects of the loss of GluR4A on OPC migration, which may be a result of reduced neuronal signaling and subsequent reduced neurotransmitter sensing by OPCs. With the uncaging of the MNI-glutamate, we directly assessed 
the ability of OPCs to sense glutamate. With this method, we created a local burst of available glutamate. In control conditions, there appeared to be two migratory gria $4 a^{+/+}$OPC populations: a slow-migrating and fast-migrating population. However, after a focal increase in glutamate availability after MNI-glutamate uncaging, we observed a shift in migration speed in these populations, with the previously slow-migrating gria $4 a^{+/+}$OPCs increasing their speed to be comparable to the fast-migrating OPCs. Almost all gria $4 a^{+/ u v a 43}$ and gria $4 a^{\text {uva } 43 / u v a 43}$ OPCs, however, were slow migrating and did not significantly increase migration speed, nor distance, in response to glutamate uncaging. We hypothesize that this lack of response to uncaged glutamate is because of the presence of the mutated GluR4A receptor. Therefore, the speed and distance of OPC migration appears to be regulated by glutamate signaling and not the inherent ability of OPCs to migrate.

\section{Regulation of myelination by AMPAR signaling}

While myelination can occur in an activity-independent manner (Barres and Raff, 1999; Bechler et al., 2018; de Faria et al., 2019), it can also be highly regulated by neuronal signaling (Barres and Raff, 1993; Hughes et al., 2013; Gautier et al., 2015; Hines et al., 2015; Mensch et al., 2015; de Faria et al., 2019). Activity-dependent myelination can be mediated by multiple mechanisms, including those involving vesicle release (Gautier et al., 2015) and NMDA receptor signaling (Lundgaard et al., 2013), and $\mathrm{Ca}^{2+}$ transients are known to stabilize myelin sheath formation and promote sheath elongation (Hines et al., 2015; Baraban et al., 2018; Krasnow et al., 2018). Because numerous interrelated pathways influence myelination, it is not surprising that we observed that $g r i a 4 a^{+/ u v a 43}$ oligodendrocytes produced less dorsal myelin than wild-type controls, as GluR4A may affect migration and myelination through distinct mechanisms. This also became apparent in our experiments with NBQX.

Previous studies have demonstrated that in rodents, AMPAR signaling regulates OPC differentiation (Gautier et al., 2015; Chen et al., 2018) and remyelination (Gautier et al., 2015). Interestingly, in vitro studies demonstrate that before the expression of MBP, there is a change in the AMPAR subunits expressed in OLCs (Itoh et al., 2002). Rat OLCs express GluR4 at an early stage, but in mature oligodendrocytes, the expression of GluR4 is decreased, hence resulting in relatively more calcium impermeable AMPAR containing GluR2.

Based on this, we hypothesize that calcium permeable AMPAR signaling enhances migration and/or blocks differentiation into mature oligodendrocytes, while calcium impermeable AMPAR signaling inhibits migration and/or stimulates differentiation. We were especially fascinated by the results obtained after treatment with NBQX, which has an opposite effect on gria $4 a^{+/+}$and gria $4 a^{\text {uva } 43 / \text { uva } 43}$ OPC migration and myelination. In gria $4 a$ mutant larvae, where there are more calcium impermeable AMPARs because of a mutation in gria $4 a$ and increased expression of gria $2 b$, OPC migration, differentiation, and myelination are regulated differently than in wild-type larvae. Because NBQX is a generic AMPAR antagonist, it can inhibit all AMPA subunits. In wild-type larvae, NBQX treatment from 30 to $72 \mathrm{hpf}$ would block GluR4A function, thus blocking OPC migration. However, in our gria $4 a^{\text {uva } 3 / \text { uva } 43}$ larvae, NBQX treatment from 30 to $72 \mathrm{hpf}$ would also have a significant block of GluR2B function because of the increased expression levels of this subunit, which could therefore, result in increased migration and also affect myelination. These data demonstrate that fine-tuned, temporal control of expression of distinct AMPAR subunits is required for both efficient dorsal OPC migration and myelination, and that this balance between calcium-permeable and calcium-impermeable subunits determines when OPCs migrate and differentiate.

Downstream of glutamate sensing, $\mathrm{Ca}^{2+}$ influx drives OPC migration and initiation of differentiation, as evidenced by the rescued OPC migration and myelination in gria $4 a^{\text {uva } 43 / \text { uva } 43}$ embryos treated with L-type voltage-gated $\mathrm{Ca}^{2+}$ channel agonist (士)-Bay K 8644. This is in line with a previous studies that revealed that voltage-operated $\mathrm{Ca}^{2+}$ channels affect OPC migration and that voltage-gated $\mathrm{Ca}^{2+}$ influx in oligodendroglial cells is critical for normal myelination (Cheli et al., 2016) and $\mathrm{Ca}^{2+}$ transients are known to stabilize myelin sheath formation and promote sheath elongation (Hines et al., 2015; Baraban et al., 2018; Krasnow et al., 2018). In another study using primary cultures of mouse OPCs, L-type voltage gated channel signaling increased OPC morphologic differentiation as well as the expression of mature oligodendrocyte markers (Cheli et al., 2015). In our work, we do not observe any increase in the number of myelin internodes or the average internode length in response to (士)-Bay K 8644 in gria $4 a^{+/+}$larvae, and the average myelin internode length in gria $4 a^{+/ 4 v a 43}$ larvae even slightly decreased. Interestingly, a recent study in zebrafish demonstrated that some OPCs participate extensively in signaling involving $\mathrm{Ca}^{2+}$ transients, but preferentially proliferate rather than differentiate into myelin-producing cells (Marisca et al., 2020). However, in our study we did observe a positive effect of ( \pm )-Bay K 8644 on myelination in gria $4 a^{\text {uva } 43 / \text { uva } 43}$ larvae. Notably, while OPC migration in gria $4 a^{\text {uva } 43 / \text { uva }}$ larvae is restored after $16 \mathrm{~h}$ of voltage-gated calcium channel activation with ( \pm )-Bay $\mathrm{K} 8644$, there is only a rescue of myelination on a 24-h treatment with ( \pm )-Bay K 8644. Therefore, is seems that $\mathrm{Ca}^{2+}$ influx via voltage-gated calcium channels is especially important for initiation of myelination.

\section{AMPAR signaling affects multiple processes in OLCs}

The effects of inhibiting AMPAR signaling in OLCs are contextspecific and diverse. One recent study reported that an induced, mature oligodendrocyte-specific knock-out of gria4 in mice ameliorated EAE symptoms by reducing oligodendrocyte vulnerability to excitotoxicity (Evonuk et al., 2020). In multiple sclerosis (MS), insufficient migration of OPCs to lesions results in poor remyelination (Boyd et al., 2013). NBQX inhibits the early phases of remyelination in vivo in toxin-induced demyelinated lesions in the rat brain, which was attributed to a direct effect on OPCs (Gautier et al., 2015). Inhibition of neuronal activity increased the numbers of OPCs in these demyelinated lesions, while OPC differentiation was reduced (Gautier et al., 2015). Additionally, the loss of GluR4 in rat brain inhibits OPC survival (Kougioumtzidou et al., 2017). Our findings show that GluR4A AMPAR activation drives OPC migration, which raises the question of whether GluR4A-deficient zebrafish would have enhanced remyelination capacity because of reduced vulnerability to excitotoxicity, or would fail to remyelinate MS-like lesions because of a defect in OPC migration, as seen in early myelination of the dorsal spinal cord. The expression of AMPAR subunits in OLCs is tightly regulated; changing one subunit can control migration, proliferation, differentiation, and/or myelination depending on the spatiotemporal environment, which makes OPC AMPAR signaling an exciting area for future research.

\section{References}

Almeida RG, Czopka T, Ffrench-Constant C, Lyons DA (2011) Individual axons regulate the myelinating potential of single oligodendrocytes in vivo. Development 138:4443-4450. 
Baraban M, Koudelka S, Lyons DA (2018) Ca 2+ activity signatures of myelin sheath formation and growth in vivo. Nat Neurosci 21:19-23.

Barres BA, Raff MC (1993) Proliferation of oligodendrocyte precursor cells depends on electrical activity in axons. Nature 361:258-260.

Barres BA, Raff MC (1999) Axonal control of oligodendrocyte development. J Cell Biol 147:1123-1128.

Bechler ME, Swire M, Ffrench-Constant C (2018) Intrinsic and adaptive myelination-A sequential mechanism for smart wiring in the brain. Dev Neurobiol 78:68-79.

Bergles DE, Roberts JD, Somogyi P, Jahr CE (2000) Glutamatergic synapses on oligodendrocyte precursor cells in the hippocampus. Nature 405:187191.

Binari LA, Lewis GM, Kucenas S (2013) Perineurial glia require notch signaling during motor nerve development but not regeneration. J Neurosci 33:4241-4252.

Blankenfeld Gv G. v, Verkhratsky AN, Kettenmann H (1992) Ca2 + channel expression in the oligodendrocyte lineage. Eur J Neurosci 4:1035-1048.

Boyd A, Zhang H, Williams A (2013) Insufficient OPC migration into demyelinated lesions is a cause of poor remyelination in MS and mouse models. Acta Neuropathol 125:841-859.

Burnashev N (1998) Calcium permeability of ligand-gated channels. Cell Calcium 24:325-332.

Butt AM (2006) Neurotransmitter-mediated calcium signalling in oligodendrocyte physiology and pathology. Glia 54:666-675.

Carney TJ, Dutton KA, Greenhill E, Delfino-Machín M, Dufourcq P, Blader P, Kelsh RN (2006) A direct role for Sox10 in specification of neural crest-derived sensory neurons. Development 133:4619-4630.

Cheli VT, Santiago González DA, Spreuer V, Paez PM (2015) Voltage-gated $\mathrm{Ca}++$ entry promotes oligodendrocyte progenitor cell maturation and myelination in vitro. Exp Neurol 265:69-83.

Cheli VT, Santiago González DA, Namgyal Lama T, Spreuer V, Handley V, Murphy GG, Paez PM (2016) Conditional deletion of the L-type calcium channel Cav1.2 in oligodendrocyte progenitor cells affects postnatal myelination in mice. J Neurosci 36:10853-10869.

Chen TJ, Kula B, Nagy B, Barzan R, Gall A, Ehrlich I, Kukley M (2018) In vivo regulation of oligodendrocyte precursor cell proliferation and differentiation by the AMPA-receptor subunit GluA2. Cell Rep 25:852-861.e7.

Danesi C, Keinänen K, Castrén ML (2019) Dysregulated Ca2+-permeable AMPA receptor signaling in neural progenitors modeling fragile $\mathrm{X}$ syndrome. Front Synaptic Neurosci 11:2.

de Faria O, Gonsalvez DG, Nicholson M, Xiao J (2019) Activity-dependent central nervous system myelination throughout life. J Neurochem 148:447-461.

Deng W, Rosenberg PA, Volpe JJ, Jensen FE (2003) Calcium-permeable AMPA/kainate receptors mediate toxicity and preconditioning by oxygen-glucose deprivation in oligodendrocyte precursors. Proc Natl Acad Sci USA 100:6801-6806.

Emery B (2010) Regulation of oligodendrocyte differentiation and myelination. Science 330:779-782.

Evonuk KS, Doyle RE, Moseley CE, Thornell IM, Adler K, Bingaman AM, Bevensee MO, Weaver CT, Min B, DeSilva TM (2020) Reduction of AMPA receptor activity on mature oligodendrocytes attenuates loss of myelinated axons in autoimmune neuroinflammation. Sci Adv 6: eaax5936.

Feldmeyer D, Kask K, Brusa R, Kornau HC, Kolhekar R, Rozov A, Burnashev N, Jensen V, Hvalby O, Sprengel R, Seeburg PH (1999) Neurological dysfunctions in mice expressing different levels of the $Q / R$ site-unedited AMPAR subunit GluR-B. Nat Neurosci 2:57-64.

Fontenas L, Welsh TG, Piller M, Coughenour P, Gandhi AV, Prober DA, Kucenas S (2019) The neuromodulator adenosine regulates oligodendrocyte migration at motor exit point transition zones. Cell Rep 27:115-128.e5.

Frost EE, Zhou Z, Krasnesky K, Armstrong RC (2009) Initiation of oligodendrocyte progenitor cell migration by a PDGF-A activated extracellular regulated kinase (ERK) signaling pathway. Neurochem Res 34:169-181.

Gagnon JA, Valen E, Thyme SB, Huang P, Ahkmetova L, Pauli A, Montague TG, Zimmerman S, Richter C, Schier AF (2014) Efficient mutagenesis by Cas9 protein-mediated oligonucleotide insertion and large-scale assessment of single-guide RNAs. PLoS One 9:e98186.

Gallo V, Armstrong RC (2008) Myelin repair strategies: a cellular view. Curr Opin Neurol 21:278-283.
Gansner JM, Dang M, Ammerman M, Zon LI (2017) Transplantation in zebrafish. Methods Cell Biol 138:629-647.

Gautier HOB, Evans KA, Volbracht K, James R, Sitnikov S, Lundgaard I, James F, Lao-Peregrin C, Reynolds R, Franklin RJM, Káradóttir RT (2015) Neuronal activity regulates remyelination via glutamate signalling to oligodendrocyte progenitors. Nat Commun 6:8518.

Ge WP, Yang XJ, Zhang Z, Wang HK, Shen W, Deng QD, Duan S (2006) Long-term potentiation of neuron-glia synapses mediated by $\mathrm{Ca} 2+$-permeable AMPA receptors. Science 312:1533-1537.

Gibson EM, Purger D, Mount CW, Goldstein AK, Lin GL, Wood LS, Inema I, Miller SE, Bieri G, Zuchero JB, Barres BA, Woo PJ, Vogel H, Monje M (2014) Neuronal activity promotes oligodendrogenesis and adaptive myelination in the mammalian brain. Science 344:1252304.

Giesen J, Füchtbauer E-M, Füchtbauer A, Funke K, Koesling D, Russwurm M (2020) AMPA induces NO-dependent cGMP signals in hippocampal and cortical neurons via L-type voltage-gated calcium channels. Cereb Cortex 30:2128-2143.

Gudz TI, Komuro H, Macklin WB (2006) Glutamate stimulates oligodendrocyte progenitor migration mediated via an alphav integrin/myelin proteolipid protein complex. J Neurosci 26:2458-2466.

Harlow DE, Saul KE, Komuro H, Macklin WB (2015) Myelin proteolipid protein complexes with $\alpha \mathrm{v}$ integrin and AMPA receptors in vivo and regulates AMPA-dependent oligodendrocyte progenitor cell migration through the modulation of cell-surface GluR2 expression. J Neurosci 35:12018-12032.

Higuchi M, Maas S, Single FN, Hartner J, Rozov A, Burnashev N, Feldmeyer D, Sprengel R, Seeburg PH (2000) Point mutation in an AMPA receptor gene rescues lethality in mice deficient in the RNA-editing enzyme ADAR2. Nature 406:78-81.

Hines JH, Ravanelli AM, Schwindt R, Scott EK, Appel B (2015) Neuronal activity biases axon selection for myelination in vivo. Nat Neurosci 18:683689.

Hochgreb-Hägele T, Bronner ME (2013) A novel FoxD3 gene trap line reveals neural crest precursor movement and a role for FoxD3 in their specification. Dev Biol 374:1-11.

Hoppmann V, Wu JJ, Søviknes AM, Helvik JV, Becker TS (2008) Expression of the eight AMPA receptor subunit genes in the developing central nervous system and sensory organs of zebrafish. Dev Dyn 237:788-799.

Hughes AN, Appel B (2020) Microglia phagocytose myelin sheaths to modify developmental myelination. Nat Neurosci 23:1055-1066.

Hughes EG, Kang SH, Fukaya M, Bergles DE (2013) Oligodendrocyte progenitors balance growth with self-repulsion to achieve homeostasis in the adult brain. Nat Neurosci 16:668-676.

Isaac JTR, Ashby MC, McBain CJ (2007) The role of the GluR2 subunit in AMPA receptor function and synaptic plasticity. Neuron 54:859-871.

Itoh T, Beesley J, Itoh A, Cohen AS, Kavanaugh B, Coulter DA, Grinspan JB, Pleasure D (2002) AMPA glutamate receptor-mediated calcium signaling is transiently enhanced during development of oligodendrocytes. J Neurochem 81:390-402.

Karlsson J, von Hofsten J, Olsson PE (2001) Generating transparent zebrafish: a refined method to improve detection of gene expression during embryonic development. Mar Biotechnol (NY) 3:522-527.

Kawakami K (2004) Transgenesis and gene trap methods in zebrafish by using the Tol2 transposable element. Methods Cell Biol 77:201-222.

Kimmel CB, Ballard WW, Kimmel SR, Ullmann B, Schilling TF (1995) Stages of embryonic development of the zebrafish. Dev Dyn 203:253310.

Kirby BB, Takada N, Latimer AJ, Shin J, Carney TJ, Kelsh RN, Appel B (2006) In vivo time-lapse imaging shows dynamic oligodendrocyte progenitor behavior during zebrafish development. Nat Neurosci 9:15061511.

Koleilat A, Dugdale JA, Christenson TA, Bellah JL, Lambert AM, Masino MA, Ekker SC, Schimmenti LA (2019) L-type voltage-gated calcium channel agonists improve hearing loss and modify ribbon synapse morphology in the zebrafish model of Usher syndrome type 1. bioRxiv. doi: 10.1101/2019.12.16.878231.

Koudelka S, Voas MG, Almeida RG, Baraban M, Soetaert J, Meyer MP, Talbot WS, Lyons DA (2016) Individual neuronal subtypes exhibit diversity in CNS myelination mediated by synaptic vesicle release. Curr Biol 26:1447-1455.

Kougioumtzidou E, Shimizu T, Hamilton NB, Tohyama K, Sprengel R, Monyer H, Attwell D, Richardson WD (2017) Signalling through AMPA 
receptors on oligodendrocyte precursors promotes myelination by enhancing oligodendrocyte survival. Elife 6:e28080.

Krasnow AM, Ford MC, Valdivia LE, Wilson SW, Attwell D (2018) Regulation of developing myelin sheath elongation by oligodendrocyte calcium transients in vivo. Nat Neurosci 21:24-28.

Kucenas S, Snell H, Appel B (2008) Nkx2.2a promotes specification and differentiation of a myelinating subset of oligodendrocyte lineage cells in zebrafish. Neuron Glia Biol 4:71.

Kukley M, Capetillo-Zarate E, Dietrich D (2007) Vesicular glutamate release from axons in white matter. Nat Neurosci 10:311-320.

Kula B, Chen TJ, Kukley M (2019) Glutamatergic signaling between neurons and oligodendrocyte lineage cells: is it synaptic or non-synaptic? Glia 67:2071-2091.

Kung SS, Chen YC, Lin WH, Chen CC, Chow WY (2001) Q/R RNA editing of the AMPA receptor subunit 2 (GRIA2) transcript evolves no later than the appearance of cartilaginous fishes. FEBS Letters 509:277-281.

Kwan KM, Fujimoto E, Grabher C, Mangum BD, Hardy ME, Campbell DS, Parant JM, Yost HJ, Kanki JP, Chien C-B (2007) The Tol2kit: a multisite gateway-based construction kit for Tol2 transposon transgenesis constructs. Dev Dyn 236:3088-3099.

Labun K, Guo X, Chavez A, Church G, Gagnon JA, Valen E (2018) Accurate analysis of genuine CRISPR editing events with ampliCan. bioRxiv 249474.

Lee CJ, Kong H, Manzini MC, Albuquerque C, Chao MV, MacDermott AB (2001) Kainate receptors expressed by a subpopulation of developing nociceptors rapidly switch from high to low $\mathrm{Ca} 2+$ permeability. J Neurosci 21:4572-4581.

Li Y, Du X, Liu C, Wen Z, Du J (2012) Reciprocal regulation between resting microglial dynamics and neuronal activity in vivo. Dev Cell 23:11891202.

Lin S, Bergles DE (2004) Synaptic signaling between GABAergic interneurons and oligodendrocyte precursor cells in the hippocampus. Nat Neurosci 7:24-32.

Love MI, Huber W, Anders S (2014) Moderated estimation of fold change and dispersion for RNA-seq data with DESeq2. Genome Biol 15:550.

Lundgaard I, Luzhynskaya A, Stockley JH, Wang Z, Evans KA, Swire M, Volbracht K, Gautier HOB, Franklin RJM, ffrench-Constant C, Attwell D, Káradóttir RT (2013) Neuregulin and BDNF induce a switch to NMDA receptor-dependent myelination by oligodendrocytes. PLoS Biol 11:e1001743.

Marisca R, Hoche T, Agirre E, Hoodless LJ, Barkey W, Auer F, CasteloBranco G, Czopka T (2020) Functionally distinct subgroups of oligodendrocyte precursor cells integrate neural activity and execute myelin formation. Nat Neurosci 23:363-374.

Marvin JS, Scholl B, Wilson DE, Podgorski K, Kazemipour A, Müller JA, Schoch S, Quiroz FJU, Rebola N, Bao H, Little JP, Tkachuk AN, Cai E, Hantman AW, Wang SSH, DePiero VJ, Borghuis BG, Chapman ER, Dietrich D, et al. (2018) Stability, affinity, and chromatic variants of the glutamate sensor iGluSnFR. Nat Methods 15:936-939.

Mensch S, Baraban M, Almeida R, Czopka T, Ausborn J, El Manira A, Lyons DA (2015) Synaptic vesicle release regulates myelin sheath number of individual oligodendrocytes in vivo. Nat Neurosci 18:628-630.

Meyer A, Schartl M (1999) Gene and genome duplications in vertebrates: the one-to-four (-to-eight in fish) rule and the evolution of novel gene functions. Curr Opin Cell Biol 11:699-704.

Mitew S, Hay CM, Peckham H, Xiao J, Koenning M, Emery B (2014) Mechanisms regulating the development of oligodendrocytes and central nervous system myelin. Neuroscience 276:29-47.
Nakayama T, Blitz IL, Fish MB, Odeleye AO, Manohar S, Cho KWY, Grainger RM (2014) Cas9-based genome editing in Xenopus tropicalis. Methods Enzymol 546:355-375.

Nave KA, Werner HB (2014) Myelination of the nervous system: mechanisms and functions. Annu Rev Cell Dev Biol 30:503-533.

Noori R, Park D, Griffiths JD, Bells S, Frankland PW, Mabbott D, Lefebvre J (2020) Activity-dependent myelination: a glial mechanism of oscillatory self-organization in large-scale brain networks. Proc Natl Acad Sci USA 117:13227-13237.

Paez PM, Fulton DJ, Spreur V, Handley V, Campagnoni AT (2010) Multiple kinase pathways regulate voltage-dependent $\mathrm{Ca} 2+$ influx and migration in oligodendrocyte precursor cells. J Neurosci 30:6422-6433.

Pagès H, Carlson M, Falcon S, Li N (2020) AnnotationDbi: manipulation of SQLite-based annotations in bioconductor. R package version 1.52.0. Available at https://bioconductor.org/packages/AnnotationDbi.

Peri F, Nüsslein-Volhard C (2008) Live imaging of neuronal degradation by microglia reveals a role for v0-ATPase al in phagosomal fusion in vivo. Cell 133:916-927.

Peterson SM, Freeman JL (2009) RNA isolation from embryonic zebrafish and cDNA synthesis for gene expression analysis. J Vis Exp. Advance online publication. Retrieved Aug 7, 2009. doi: 10.3791/1470.

Sanchez-Rodriguez MA, Gomez O, Esteban PF, Garcia-Ovejero D, MolinaHolgado E (2018) The endocannabinoid 2-arachidonoylglycerol regulates oligodendrocyte progenitor cell migration. Biochem Pharmacol 157:180188.

Shin J, Park H-C, Topczewska JM, Mawdsley DJ, Appel B (2003) Neural cell fate analysis in zebrafish using olig2 BAC transgenics. Methods Cell Sci 25:7-14.

Smith CJ, Morris AD, Welsh TG, Kucenas S (2014) Contact-mediated inhibition between oligodendrocyte progenitor cells and motor exit point glia establishes the spinal cord transition zone. PLoS Biol 12:e1001961.

Sobolevsky AI, Yelshansky MV, Wollmuth LP (2003) Different gating mechanisms in glutamate receptor and K+ channels. J Neurosci 23:75597568.

Soneson C, Love MI, Robinson MD (2015) Differential analyses for RNA-seq: transcript-level estimates improve gene-level inferences. F1000Research 4:1521.

Suzuki N, Hyodo M, Hayashi C, Mabuchi Y, Sekimoto K, Onchi C, Sekiguchi K, Akazawa C (2019) Laminin $\alpha 2, \alpha 4$, and $\alpha 5$ chains positively regulate migration and survival of oligodendrocyte precursor cells. Sci Rep 9:19882.

Wake H, Ortiz FC, Woo DH, Lee PR, Angulo MC, Fields RD (2015) Nonsynaptic junctions on myelinating glia promote preferential myelination of electrically active axons. Nat Commun 6:7844.

Yu G, Wang LG, Han Y, He QY (2012) clusterProfiler: an R package for comparing biological themes among gene clusters. OMICS 16:284-287.

Yuan X, Eisen AM, McBain CJ, Gallo V (1998) A role for glutamate and its receptors in the regulation of oligodendrocyte development in cerebellar tissue slices. Development 125:2901-2914.

Zhu Y, Crowley SC, Latimer AJ, Lewis GM, Nash R, Kucenas S (2019) Migratory neural crest cells phagocytose dead cells in the developing nervous system. Cell 179:74-89.e10.

Ziskin JL, Nishiyama A, Rubio M, Fukaya M, Bergles DE (2007) Vesicular release of glutamate from unmyelinated axons in white matter. Nat Neurosci 10:321-330 